Check for updates

Cite this: RSC Adv., 2018, 8, 5702

Received 10th December 2017 Accepted 19th January 2018

DOI: $10.1039 / c 7 r a 13207 g$

rsc.li/rsc-advances

\title{
Green synthesis of new pyrrolidine-fused spirooxindoles via three-component domino reaction in EtOH/ $\mathrm{H}_{2} \mathrm{O} \uparrow$
}

\author{
Yong-Chao Wang, (DD *a Jun-Liang Wang, ${ }^{c}$ Kevin S. Burgess, ${ }^{d}$ Jiang-Wei Zhang, ${ }^{e}$ \\ Qiu-Mei Zheng, ${ }^{a}$ Ya-Dan Pu, ${ }^{a}$ Li-Jun Yan ${ }^{\star a}$ and Xue-Bing Chen iD *b
}

An efficient, green and sustainable approach for the synthesis of novel polycyclic pyrrolidine-fused spirooxindole compounds was developed. The synthesis included a one-pot, three-component, domino reaction of $(E)$-3-(2-nitrovinyl)-indoles, isatins and chiral polycyclic $\alpha$-amino acids under catalyst-free conditions at room temperature in $\mathrm{EtOH}-\mathrm{H}_{2} \mathrm{O}$. The salient features of this methodology are ecofriendliness, high yields and the ease of obtaining target compounds without the involvement of toxic solvents and column chromatography. These novel polycyclic pyrrolidine-fused spirooxindoles provide a collection of structurally diverse compounds that show promise for future bioassays and medical treatments.

\section{Introduction}

To adhere to the principles of "green chemistry" and "benign by design", ${ }^{1}$ there is an immediate need to develop efficient chemical synthetic strategies that are environmentally friendly, sustainable, and atom-economical. To maximize reaction sustainability and safety, ${ }^{2}$ development of novel synthetic strategies that involve greener reaction media have resulted in sustainable pathways for chemical synthesis. ${ }^{3}$ An alcohol-water solution, as a nontoxic, inexpensive and widely available solvent, has been shown to not only accelerate the rate of organic reactions, even for water-insoluble reactants, but can also simplify purification operations to standard filtration or recrystallization. ${ }^{4}$ Recently, multicomponent reactions (MCRs) ${ }^{5}$ have emerged as efficient tools for synthesizing functionally and chemically diverse novel heterocyclic compounds in organic and medicinal chemistry. Of immediate need is the

${ }^{a}$ School of Vocational and Technical Education, Yunnan Normal University, Kunming 650092, PR China. E-mail: yongchaowang126@126.com; yanlijunhappy@126.com; Tel: +8615925166595

${ }^{b}$ Key Laboratory of Natural Pharmaceutical and Chemical Biology of Yunnan Province, School of Science, Honghe University, Mengzi, Yunnan, 661199, PR China. E-mail: orangekaka@126.com

${ }^{c}$ School of Chemical Science and Technology, Yunnan University, Kunming 650091, PR China

${ }^{d}$ Department of Biology, College of Letters \& Sciences, Columbus State University, University System of Georgia, Columbus, GA, USA

${ }^{e}$ Gold Catalysis Research Center, State Key Laboratory of Catalysis, Dalian Institute of Chemical Physics, Chinese Academy of Sciences, Dalian 116023, PR China

$\dagger$ Electronic supplementary information (ESI) available: ${ }^{1} \mathrm{H}$ and ${ }^{13} \mathrm{C}$ NMR spectra for compounds 5a-5e, 4a-4w, 8a-81. CCDC 1817780. For ESI and crystallographic data in CIF or other electronic format see DOI: $10.1039 / \mathrm{c} 7 \mathrm{ra13207g}$ exploration of new MCRs in alcohol-water solution for the organic synthesis of novel compounds for drug discovery and the promotion of green chemistry. ${ }^{6}$

Spirooxindole ring systems are frequently encountered in natural alkaloids ${ }^{7}$ and are often considered as attractive templates for drug discovery. ${ }^{8}$ Natural and synthetic alkaloids containing an indole moiety exhibit a wide spectrum of biological activities including anti-tumor, ${ }^{9}$ anti-microbial, ${ }^{10}$ anti-malarial, ${ }^{11}$ anti-diabetic, ${ }^{12}$ anti-tubercular, ${ }^{13}$ anti-HIV ${ }^{14}$ anti-oxidant ${ }^{15}$ and other biological activities. ${ }^{16}$ Their remarkable pharmacological activity and unique molecular architecture have made spirooxindoles, and their derivatives, attractive synthetic targets. ${ }^{17}$ In addition, functionalized polycyclic $N$-fused-pyrrolidines have also been shown to have a wide spectrum of biological activities that include anti-tumor, anti-HIV and other anti-viral disease activities. ${ }^{18}$ Spiro-fused cyclic frameworks are known to be the central skeletons of numerous alkaloids and pharmacologically important compounds with various types of bioactivities. ${ }^{19}$ The two organic frameworks are regarded as templates for drug discovery and scaffolds for combinatorial libraries, respectively, ${ }^{20}$ the presence of two or more different heterocyclic moieties in a single molecule could remarkably enhance biological activity. ${ }^{21}$ To date, some representative molecules (1-3) have been designed and synthesized, which exhibit outstanding pharmacological activities $^{22}$ (Fig. 1). Here we speculate that the integration of spirooxindole, polycyclic $N$-fused-pyrrolidines and spiro-fused cyclic frameworks into a molecule may result in the discovery of new drug candidates. Polycyclic pyrrolidine-fused spirooxindoles may exhibit a wide range of useful pharmacological properties and biological activities in combination with the pharmacological activity of spirooxindole, $N$-fused-pyrrolidines and spiro-fused cyclic frameworks. 


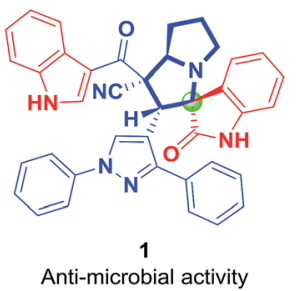

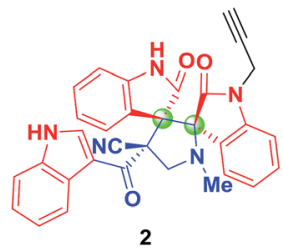

Anti-cancer and anti-microbial activities

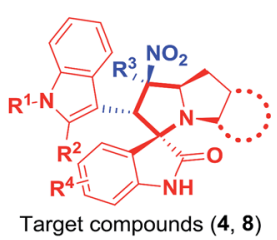

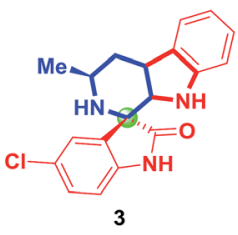

Anti-malarial activity NF54 I $\mathrm{C}_{50}=9 \mathrm{nM}$
Fig. 1 Selected representative biologically active heterocycles and target compounds that exhibit pharmacological activities.

Despite recent attention on the synthesis of novel spirocyclic oxindoles, the development of green and sustainable methods to access spirooxindoles remains challenging. Only a limited number of methodologies have been reported for the synthesis of the above-mentioned compounds, ${ }^{23}$ and most require toxic organic solvents, harsh reaction conditions, expensive catalysts or involve complex separation processes. The development of a milder, more eco-friendly and efficient method for synthesizing combined novel spirooxindoles under aqueous solvent conditions is necessary. Here, we described a relatively easy and green method for the synthesis of novel polycyclic pyrrolidinefused spirooxindole derivatives $(\mathbf{4}, \mathbf{8})$ (Fig. 1) using a catalystfree, one-pot three-component reaction of $(E)$-3-(2-nitrovinyl)indoles (5), isatins (6) and polycyclic chiral $\alpha$-amino acids (7) in alcohol-water solution at room temperature. To the best of our knowledge, this is the first green synthesis of a heterocyclic compound 4.

\section{Results and discussion}

In this study, we report a green method for the synthesis of novel polycyclic pyrrolidine-fused spirooxindole derivatives (4) via one-pot three-component domino reaction of $(E)$-3-(2-nitrovinyl)- $1 H$-indoles (5), isatins (6) and polycyclic chiral $\alpha$-amino acids (7) using an alcohol-water solution as a green medium at room temperature.

To establish the feasibility of this strategy as well as to optimize the reaction conditions, the three-component reaction of (E)-1-methyl-3-(2-nitrovinyl)- $1 \mathrm{H}$-indole (5a, $1.0 \mathrm{mmol})$, isatin $(6 \mathbf{a}, \quad 1.1 \mathrm{mmol})$ and $(2 S, 3 \mathrm{a}, 7 \mathrm{a} S)$-octahydro- $1 H$-indole-2carboxylic acid (7a, $1.2 \mathrm{mmol})$ was selected as the model reaction. Initially, the model reaction was performed in a range of organic solvents at room temperature for $6 \mathrm{~h}$ of stirring (Table 1, entries 1-14). The model reaction barely proceeded when acetone, acetonitrile, toluene and diethyl ether were used as reaction solvents, respectively (Table 1, entries 1-4). Conversely, the model reaction proceeded faster with moderate (38-81\%) to high (91-92\%) yields when other (non-)low-polar solvents $\left(\mathrm{CH}_{2} \mathrm{Cl}_{2}, \mathrm{CHCl}_{3}\right)$ and polar solvents
Table 1 Optimization of the reaction conditions for the model reaction ${ }^{a}$

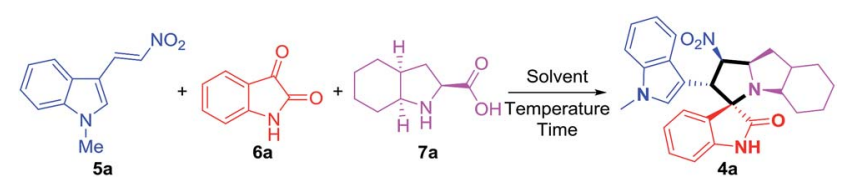

\begin{tabular}{lllll}
\hline Entry & Solvent & Temperature & Time $(\mathrm{h})$ & Yield $^{b}(\%)$ \\
\hline 1 & Acetone & r.t. & 6 & $<5$ \\
2 & Acetonitrile & r.t. & 6 & $<5$ \\
3 & Toluene & r.t. & 6 & $<5$ \\
4 & Diethyl ether & r.t. & 6 & $<5$ \\
5 & THF & r.t. & 6 & 38 \\
6 & $1,4-\mathrm{Dioxane}$ & r.t. & 6 & 41 \\
7 & $\mathrm{CH}_{2} \mathrm{Cl}_{2}$ & r.t. & 6 & 78 \\
8 & $\mathrm{CHCl}_{3}$ & r.t. & 6 & 81 \\
9 & $\mathrm{DMSO}$ & r.t. & 6 & 74 \\
10 & $\mathrm{DMF}$ & r.t. & 6 & 69 \\
11 & IsO-propanol & r.t. & 6 & 76 \\
12 & $\mathrm{Glycerol}$ & r.t. & 6 & 57 \\
13 & $\mathrm{MeOH}$ & r.t. & 6 & 91 \\
14 & EtOH & r.t. & 6 & 92 \\
15 & $\mathrm{H}_{2} \mathrm{O}$ & r.t. & 6 & 21 \\
$16^{d}$ & EtOH $/ \mathrm{H}_{2} \mathrm{O}=1: 1$ & r.t. & 6 & 94 \\
$17^{d}$ & EtOH $/ \mathrm{H}_{2} \mathrm{O}=1: 2$ & r.t. & 6 & 87 \\
$18^{d}$ & $\mathrm{EtOH} / \mathrm{H}_{2} \mathrm{O}=1: 1$ & $50{ }^{\circ} \mathrm{C}$ & 6 & 95 \\
$19^{d}$ & EtOH $/ \mathrm{H}_{2} \mathrm{O}=1: 1$ & Reflux & 6 & 95 \\
$20^{d}$ & EtOH$/ \mathrm{H}_{2} \mathrm{O}=1: 1$ & r.t. & 12 & 95
\end{tabular}

${ }^{a}$ All reactions were carried out with $5 \mathrm{a}(1.0 \mathrm{mmol}), 6 \mathrm{a}(1.1 \mathrm{mmol})$ and $7 \mathrm{a}$ $(1.2 \mathrm{mmol})$ in corresponding solvents $(5.0 \mathrm{~mL})$ at corresponding temperatures. ${ }^{b}$ Isolated yields based on $\beta$-nitrostyrene (5a). ${ }^{c}$ Room temperature. ${ }^{d}$ The resulting precipitates were filtered and washed with 3-5 $\mathrm{mL} \mathrm{EtOH} / \mathrm{H}_{2} \mathrm{O}(\mathrm{v} / \mathrm{v}=1: 1)$.

(THF, 1,4-dioxane, DMSO, DMF, iso-propanol, glycerol, $\mathrm{MeOH}$ and EtOH) were screened (Table 1, entries 5-14): the highest yield was achieved when EtOH was used as a solvent (Table 1, entry $14,92 \%$ yield). Interestingly, the desired product was also obtained in water medium (Table 1, entry 15) although the yield was only $21 \%$. The low yield in water medium could be attributed to the poor dissolution of raw materials since almost all the unreacted starting material could been recycled and reused.

Based on a comprehensive assessment of these results, we chose an ethanol-water solution as the optimal reaction solvent, which we then optimized to further explore a more eco-friendly synthetic condition (Table 1, entries 16 and 17). Among the different proportions of EtOH/ $\mathrm{H}_{2} \mathrm{O}$ solvents surveyed, EtOH/ $\mathrm{H}_{2} \mathrm{O}(1: 1)$ was found to be the most suitable solvent for the model reaction ( $94 \%$ yield, Table 1, entry 16 ). To maximize the product yield, we then tested the best reaction temperature (Table 1, entries 16,18-19). Yields were not significantly different when the reaction temperature was increased from room temperature to $50{ }^{\circ} \mathrm{C}$ or to reflux (94\% vs. $95 \%$ vs. $95 \%$ yields, respectively, Table 1, entries 16, 18-19): room temperature was the ideal reaction temperature for the synthesis of polycyclic pyrrolidine-fused spirooxindole derivative $4 \mathbf{a}$ ( $94 \%$ yield). There was no significant 
increase in yield when the reaction time was increased from $6 \mathrm{~h}$ to $12 \mathrm{~h}(95 \% v s .94 \%$ yields, respectively, entries 16,20$)$. It is worth mentioning that the highly purified target compound could be obtained by filtering the reaction precipitates and then washing with $\mathrm{EtOH} / \mathrm{H}_{2} \mathrm{O}(1: 1)$ for $2-3$ times in the absence of traditional purification techniques such as column chromatography or recrystallization. Overall, the best reaction conditions for synthesizing polycyclic pyrrolidine-fused spirooxindole compound $\mathbf{4 a}$ are achieved by employing $(E)$ 1-methyl-3-(2-nitrovinyl)-1H-indole $(5 \mathrm{a}, 1.0 \mathrm{mmol})$, isatin (6a, $1.1 \mathrm{mmol})$ and $(2 S, 3 \mathrm{a} S, 7 \mathrm{a} S)$-octahydroindole-2-carboxylic acid $(7 \mathrm{a}, 1.2 \mathrm{mmol})$ in $\mathrm{EtOH} / \mathrm{H}_{2} \mathrm{O}(1: 1)$ solvent at room temperature for $6 \mathrm{~h}$.

To explore the scope of the model reaction, we substituted various (E)-3-(2-nitrovinyl)-indoles (5) (Fig. 2) and isatin derivatives (6) (Fig. 2) as well as (2S,3aS,7aS)-octahydro- $1 H$-indole-2carboxylic acid (7a) under optimal reaction conditions. Results indicated that the desired polycyclic pyrrolidine-fused spirooxindole compounds could be obtained in relatively high yields (Table 2) from a diverse set of substrates. As shown in Table 2, (E)-3-(2-nitrovinyl)-indoles (5) bearing diverse functional groups, such as $\mathrm{H}, \mathrm{CH}_{3}$ or $\mathrm{Ph}$ group were suitable for the reaction, respectively. For the isatin derivatives (6), the aromatic ring bearing either electron-donating $\left(\mathrm{CH}_{3}, \mathrm{OCH}_{3}\right)$ or electronwithdrawing functional groups $(\mathrm{F}, \mathrm{Cl}, \mathrm{Br})$, and substitution patterns (5-substitution, 6-substitution and 7-substitution) could form the target products $4(\mathbf{4 a}-\mathbf{4 w})$ with high yields (86$95 \%)$.

To further explore the scope of the procedure reported in Table 2, the methodology was evaluated by using $(E)-3-(2-$ nitrovinyl)- $1 H$-indole (5d), isatin derivatives (6) and chiral $\alpha$-amino acids (7b) under similar conditions. The reactions proceeded smoothly and the desired polycyclic pyrrolidinefused spirooxindole compounds (8) from a diverse set of substrates were obtained (Table 3). Isatins with electron-

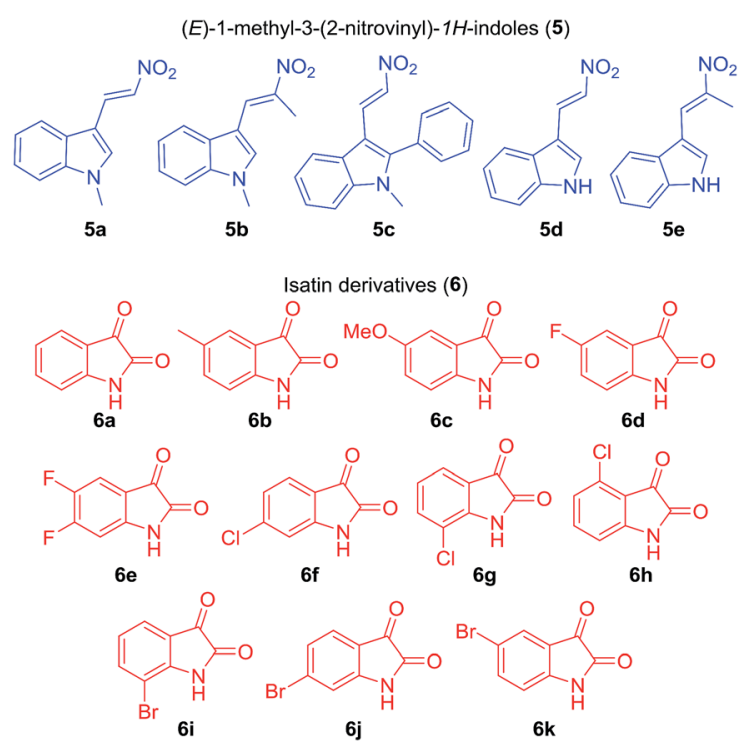

Fig. 2 The diversity of reagents (5 and 6).
Table 2 The synthesis of polycyclic pyrrolidine-fused spirooxindole derivatives $4^{a}$

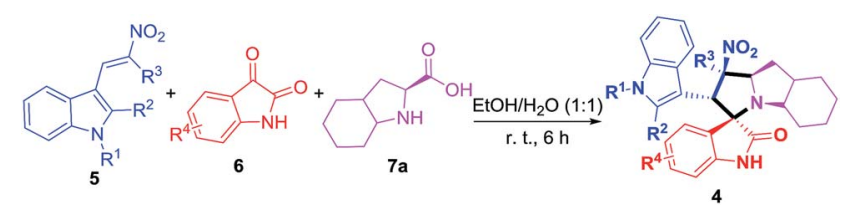

\begin{tabular}{|c|c|c|c|c|c|c|}
\hline Entry & $\mathrm{R}^{1}$ & $\mathrm{R}^{2}$ & $\mathrm{R}^{3}$ & $\mathrm{R}^{4}$ & 4 & Yield $^{b}(\%)$ \\
\hline 1 & $\mathrm{CH}_{3}$ & $\mathrm{H}$ & $\mathrm{H}$ & $\mathrm{H}$ & $4 a$ & 94 \\
\hline 2 & $\mathrm{CH}_{3}$ & $\mathrm{H}$ & $\mathrm{H}$ & $5-\mathrm{CH}_{3}$ & $4 b$ & 92 \\
\hline 3 & $\mathrm{CH}_{3}$ & $\mathrm{H}$ & $\mathrm{H}$ & $5-\mathrm{OCH}_{3}$ & $4 c$ & 91 \\
\hline 4 & $\mathrm{CH}_{3}$ & $\mathrm{H}$ & $\mathrm{H}$ & $5-\mathrm{F}$ & $4 d$ & 95 \\
\hline 5 & $\mathrm{CH}_{3}$ & $\mathrm{H}$ & $\mathrm{H}$ & 6-Cl & $4 e$ & 95 \\
\hline 6 & $\mathrm{CH}_{3}$ & $\mathrm{H}$ & $\mathrm{H}$ & $6-\mathrm{Br}$ & 4f & 96 \\
\hline 7 & $\mathrm{CH}_{3}$ & $\mathrm{H}$ & $\mathrm{H}$ & $5-\mathrm{Br}$ & $4 \mathrm{~g}$ & 94 \\
\hline 8 & $\mathrm{CH}_{3}$ & $\mathrm{H}$ & $\mathrm{CH}_{3}$ & $5-\mathrm{OCH}_{3}$ & $4 h$ & 87 \\
\hline 9 & $\mathrm{CH}_{3}$ & $\mathrm{H}$ & $\mathrm{CH}_{3}$ & $5-\mathrm{F}$ & $4 \mathbf{i}$ & 92 \\
\hline 10 & $\mathrm{CH}_{3}$ & $\mathrm{H}$ & $\mathrm{CH}_{3}$ & 5-Cl & $4 j$ & 92 \\
\hline 11 & $\mathrm{CH}_{3}$ & $\mathrm{Ph}$ & $\mathrm{H}$ & $5-\mathrm{F}$ & $4 k$ & 91 \\
\hline 12 & $\mathrm{CH}_{3}$ & $\mathrm{Ph}$ & $\mathrm{H}$ & $5-\mathrm{Br}$ & 41 & 92 \\
\hline 13 & $\mathrm{H}$ & $\mathrm{H}$ & $\mathrm{H}$ & $\mathrm{H}$ & $4 \mathrm{~m}$ & 94 \\
\hline 14 & $\mathrm{H}$ & $\mathrm{H}$ & $\mathrm{H}$ & $5-\mathrm{CH}_{3}$ & $4 n$ & 91 \\
\hline 15 & $\mathrm{H}$ & $\mathrm{H}$ & $\mathrm{H}$ & $5-\mathrm{OCH}_{3}$ & 40 & 95 \\
\hline 16 & $\mathrm{H}$ & $\mathrm{H}$ & $\mathrm{H}$ & $5-\mathrm{F}$ & $4 p$ & 92 \\
\hline 17 & $\mathrm{H}$ & $\mathrm{H}$ & $\mathrm{H}$ & 7-Cl & $4 q$ & 93 \\
\hline 18 & $\mathrm{H}$ & $\mathrm{H}$ & $\mathrm{H}$ & $5-\mathrm{Br}$ & $4 r$ & 93 \\
\hline 18 & $\mathrm{H}$ & $\mathrm{H}$ & $\mathrm{CH}_{3}$ & $5-\mathrm{CH}_{3}$ & $4 s$ & 89 \\
\hline 20 & $\mathrm{H}$ & $\mathrm{H}$ & $\mathrm{CH}_{3}$ & $5-\mathrm{OCH}_{3}$ & $4 t$ & 86 \\
\hline 21 & $\mathrm{H}$ & $\mathrm{H}$ & $\mathrm{CH}_{3}$ & $5-\mathrm{F}$ & $4 \mathbf{u}$ & 91 \\
\hline 22 & $\mathrm{H}$ & $\mathrm{H}$ & $\mathrm{CH}_{3}$ & 7-Cl & $4 v$ & 89 \\
\hline 23 & $\mathrm{H}$ & $\mathrm{H}$ & $\mathrm{CH}_{3}$ & $5-\mathrm{Br}$ & $4 w$ & 93 \\
\hline
\end{tabular}

${ }^{a}$ All reactions were carried out with $5(1.0 \mathrm{mmol}), 6(1.1 \mathrm{mmol})$ and $7 \mathrm{a}$ $(1.2 \mathrm{mmol})$ in $\mathrm{EtOH} / \mathrm{H}_{2} \mathrm{O}(\mathrm{v} / \mathrm{v}=1: 1)(5.0 \mathrm{~mL})$ at room temperatures for 6 hours. ${ }^{b}$ The resulting precipitates were filtered and washed with 3$5 \mathrm{~mL} \mathrm{EtOH} / \mathrm{H}_{2} \mathrm{O}(\mathrm{v} / \mathrm{v}=1: 1)$, the yields based on $\beta$-nitrostyrene (5).

withdrawing groups as well as electron-donating substituents underwent this one-pot conversion to give the corresponding spirooxindoles with high yields (88-96\%).

A plausible reaction mechanism was proposed (Scheme 1) based on our previous work and previous reports. ${ }^{24}$ The pyrrolidine functionality activates the carbonyl of the isatins through the formation of an enamine intermediate. Accompanying the loss of one $\mathrm{H}_{2} \mathrm{O}$ and $\mathrm{CO}_{2}$ molecule, a carbanion is produced (transition state I). Subsequently, two nucleophilic carbons then add to the corresponding electron deficient carbons of the dipolarophile during the cycloaddition via a 1,3-cycloaddition reaction (transition state $\mathbf{I I}$ ), which leads to the formation of compounds 4 and 8. From a regioselectivity perspective, the steric bulk of compounds 5 and the stability of the transition state II may explain the configuration of compounds $\mathbf{4}$ and $\mathbf{8}$. Some other typical literatures ${ }^{25}$ could be taken as a support of the regioselectivity of compounds $\mathbf{4}$ and $\mathbf{8}$. The structure deduced from NMR data and the reaction mechanism was further confirmed by X-ray analysis of a single crystal of $\mathbf{4 c}$ (Fig. 3, CCDC: 1817780†). Of note, in Fig. 3 we only presented 
Table 3 The synthesis of polycyclic pyrrolidine-fused spirooxindole derivatives $8^{a}$

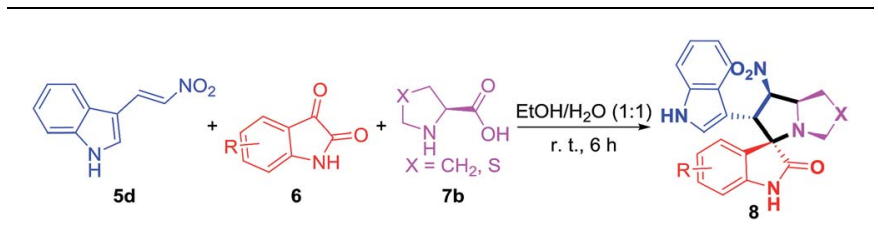

\begin{tabular}{lllll}
\hline Entry & $\mathrm{R}$ & $\mathrm{X}$ & $\mathbf{8}$ & Yield $^{b}(\%)$ \\
\hline 1 & $\mathrm{H}$ & $\mathrm{CH}_{2}$ & $\mathbf{8 a}$ & 96 \\
2 & $5-\mathrm{CH}_{3}$ & $\mathrm{CH}_{2}$ & $\mathbf{8 b}$ & 94 \\
3 & 7-CH & $\mathrm{CH}_{2}$ & $\mathbf{8 c}$ & 93 \\
4 & 5-OCH & $\mathrm{CH}_{2}$ & $\mathbf{8 d}$ & 92 \\
5 & $5-\mathrm{F}$ & $\mathrm{CH}_{2}$ & $\mathbf{8 e}$ & 93 \\
6 & $5,6-\mathrm{diF}$ & $\mathrm{CH}_{2}$ & $\mathbf{8 f}$ & 92 \\
7 & 6-Cl & $\mathrm{CH}_{2}$ & $\mathbf{8 g}$ & 95 \\
8 & 7-Cl & $\mathrm{CH}_{2}$ & $\mathbf{8 h}$ & 93 \\
9 & 6- $\mathrm{Br}$ & $\mathrm{CH}_{2}$ & $\mathbf{8 i}$ & 91 \\
10 & 7-Br & $\mathrm{CH}_{2}$ & $\mathbf{8 j}$ & 95 \\
11 & $\mathrm{H}$ & $\mathrm{S}$ & $\mathbf{8 k}$ & 89 \\
12 & 6-Cl & $\mathrm{S}$ & $\mathbf{8 1}$ & 88
\end{tabular}

${ }^{a}$ All reactions were carried out with $5 \mathbf{d}(1.0 \mathrm{mmol}), \mathbf{6}(1.1 \mathrm{mmol})$ and $7 \mathbf{b}$ $(1.2 \mathrm{mmol})$ in $\mathrm{EtOH} / \mathrm{H}_{2} \mathrm{O}(\mathrm{v} / \mathrm{v}=1: 1)(5.0 \mathrm{~mL})$ at room temperatures for 6 hours. ${ }^{b}$ The resulting precipitates were filtered and washed with 3-5 $\mathrm{mL} \mathrm{EtOH} / \mathrm{H}_{2} \mathrm{O}(\mathrm{v} / \mathrm{v}=1: 1)$, the yields based on $\beta$-nitrostyrene $(\mathbf{5 d})$.

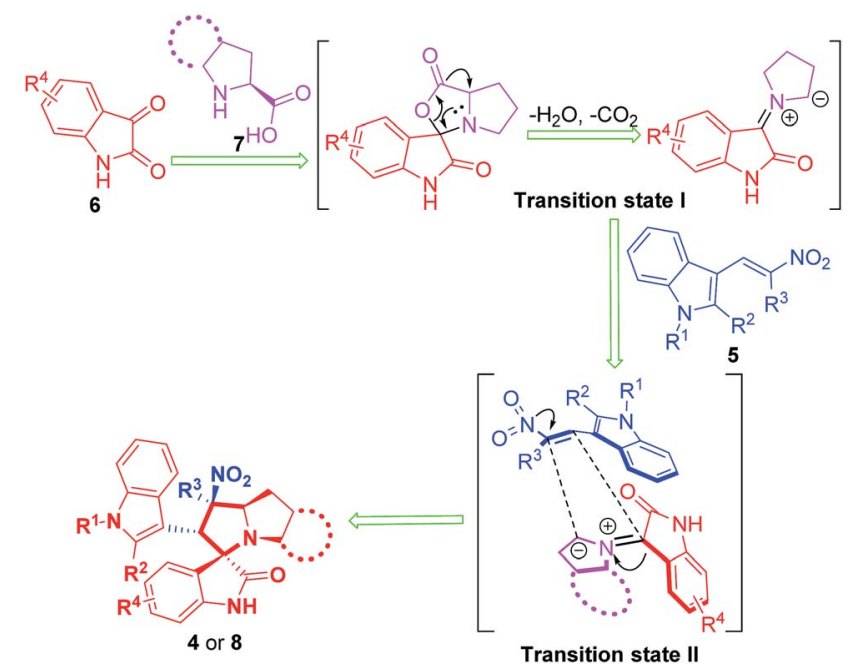

Scheme 1 Proposed reaction mechanism for the synthesis of 4 and 8 .

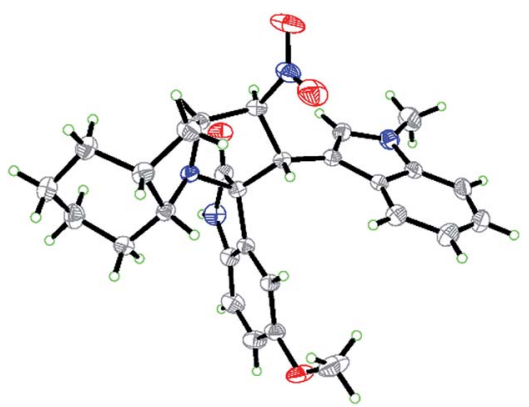

Fig. 3 Single crystal X-ray diffraction study of compound 4c. one molecule of compound $\mathbf{4 c}$ while in the single crystal structure the minimum asymmetric units contain two identical molecules of compound $\mathbf{4 c}$.

\section{Conclusions}

In this study, we developed an efficient, green and sustainable approach for the construction of multiple new $\mathrm{C}-\mathrm{C}$ bonds, $\mathrm{C}-\mathrm{N}$ bonds and polycyclic pyrrolidine-fused structure, which resulted in the synthesis of 35 novel polycyclic pyrrolidine-fused spirooxindole compounds. This synthetic method involves a one-pot, three-component domino reaction of (E)-3-(2nitrovinyl)-indoles, isatins and polycyclic chiral $\alpha$-amino acids under a catalyst-free condition at room temperature in an EtOH- $\mathrm{H}_{2} \mathrm{O}$ solution. The salient features of this methodology are environmental sustainability, high yields, ease of product purification, and the lack of toxic solvents or column chromatography during synthesis. These novel polycyclic pyrrolidinefused spirooxindoles provide a collection of promising compounds with structural diversity for future bioassays and medical treatments. In addition, the nitro group can be easily transformed into amines, amides, sulfamides, nitrile oxides and various useful functional groups for the optimization and enhancement of pharmacological activities.

\section{Experimental}

\section{General information}

Reagents and materials were of the highest commercial grade and were used without further purification. NMR spectra were recorded on a Bruker DRX $400\left({ }^{1} \mathrm{H}: 400 \mathrm{MHz},{ }^{13} \mathrm{C}: 100 \mathrm{MHz}\right)$, DRX $500\left({ }^{1} \mathrm{H}: 500 \mathrm{MHz},{ }^{13} \mathrm{C}: 125 \mathrm{MHz}\right)$ or DRX $600\left({ }^{1} \mathrm{H}: 600 \mathrm{MHz}\right.$, ${ }^{13} \mathrm{C}: 150 \mathrm{MHz}$ ) with TMS as the internal standard. Chemical shifts $(\delta)$ were expressed in ppm, $J$ values were given in $\mathrm{Hz}$, and deuterated DMSO-D $\mathrm{D}_{6}$ was used as a solvent. IR spectra were recorded on a FT-IR Thermo Nicolet Avatar 360 using KBr pellet. The mass spectroscopic data were obtained from an Agilent 1100 LC/MSD Trap LC-mass spectrometer. Melting points were determined with an XT-4A melting-point apparatus. The reactions were monitored by thin layer chromatography (TLC) with silica gel $\mathrm{GF}_{254}$, and all compounds were visualized by UV and sprayed with $\mathrm{H}_{2} \mathrm{SO}_{4}(10 \%)$ in ethanol, followed by heating. Suitable single crystal was selected. Data collections were performed by graphite-monochromated Mo-K $\alpha$ radiation $(\lambda=$ $0.71073 \AA$ A). Data reduction, cell refinement and experimental absorption correction were performed with the software package of Agilent Gemini Ultra CrysAlisPro (Ver 1.171.35.11). The structures were solved by direct methods and refined against $F^{2}$ by full-matrix least-squares. All non-hydrogen atoms were refined anisotropically. All calculations were carried out in SHELXTL ver 6.2 and Olex2 ver 1.2.9.

\section{General procedure for the synthesis of compounds $5^{26}$}

Benzene ( $18 \mu \mathrm{L}, 0.2 \mathrm{mmol})$ was titrated into a stirred solution of indole-3-carboxaldehyde (5 mmol) and $\mathrm{AcONH}_{4}(385 \mathrm{mg}$, $5 \mathrm{mmol})$ in nitromethane $(15 \mathrm{~mL})$. The mixture was stirred at 
reflux for 1-4 h. After the starting aldehyde was completely consumed (monitored by TLC), the reaction mixture was cooled to $0{ }^{\circ} \mathrm{C}$. The reaction was quenched with water and extracted with ethyl acetate $(3 \times 80 \mathrm{~mL})$. The combined organic layers were washed with saturated brine solution $(50 \mathrm{~mL})$, followed by drying with $\mathrm{Na}_{2} \mathrm{SO}_{4}$ and evaporating in vacuo. The crude product was purified by recrystallization to give the pure corresponding (E)-3-(2-nitrovinyl)-indoles (5).

(E)-1-Methyl-3-(2-nitrovinyl)-1H-indole (5a). Yellow solid; 95\% yield; mp $165-167{ }^{\circ} \mathrm{C}$; IR (KBr) 746, 791, 951, 1084, 1252, 1308, 1491, 1618, $2374 \mathrm{~cm}^{-1}$; HRMS (EI) calcd for $\mathrm{C}_{11} \mathrm{H}_{11} \mathrm{~N}_{2} \mathrm{O}_{2}$ $[\mathrm{M}+\mathrm{H}]^{+}$203.2205, found 203.2208. ${ }^{1} \mathrm{H}$ NMR (600 MHz, DMSO$\left.\mathrm{D}_{6}\right): \delta 8.35(\mathrm{~d}, J=13.8 \mathrm{~Hz}, 1 \mathrm{H}, \mathrm{CH}), 8.20(\mathrm{~s}, 1 \mathrm{H}, \operatorname{ArH}), 8.00(\mathrm{~s}, 1 \mathrm{H}$, ArH), 7.97 (d, $J=7.2 \mathrm{~Hz}, 1 \mathrm{H}, \mathrm{ArH}), 7.58$ (d, $J=8.4 \mathrm{~Hz}, 1 \mathrm{H}, \mathrm{CH})$, 7.36-7.33 (m, 1H, ArH), 7.29 (t, $J=15.0 \mathrm{~Hz}, 1 \mathrm{H}, \mathrm{ArH}), 3.86$ (s, $\left.3 \mathrm{H}, \mathrm{NCH}_{3}\right) ;{ }^{13} \mathrm{C}$ NMR (150 MHz, DMSO): $\delta$ 139.8, 138.7, 134.5, 131.5, 125.5, 123.8, 122.6, 121.0, 111.7, 107.6, 33.8.

(E)-1-Methyl-3-(2-nitroprop-1-en-1-yl)-1H-indole (5b). Yellow solid; 96\% yield; mp $136-138{ }^{\circ} \mathrm{C}$; IR (KBr) 754, 916, 972, 1126, 1236, 1283, 1470, 1531, 1634, 3124, $3297 \mathrm{~cm}^{-1}$; HRMS (EI) calcd for $\mathrm{C}_{12} \mathrm{H}_{13} \mathrm{~N}_{2} \mathrm{O}_{2}[\mathrm{M}+\mathrm{H}]^{+}$217.0972, found 217.0977. ${ }^{1} \mathrm{H}$ NMR $\left(600 \mathrm{MHz}, \mathrm{DMSO}_{\mathrm{D}}\right.$ ): $\delta 8.41$ (s, 1H, ArH), $8.01(\mathrm{~s}, 1 \mathrm{H}, \mathrm{CH}), 7.82$ (d, $J=7.8 \mathrm{~Hz}, 1 \mathrm{H}, \mathrm{ArH}), 7.54$ (d, $J=7.8 \mathrm{~Hz}, 1 \mathrm{H}, \mathrm{ArH}), 7.53-7.30$ (m, 1H, ArH), 7.25-7.23 (m, 1H, ArH), 3.89 (s, 3H, $\mathrm{NCH}_{3}$ ), 2.45 (s, $\left.3 \mathrm{H}, \mathrm{CH}_{3}\right) ;{ }^{13} \mathrm{C}$ NMR (150 MHz, DMSO): $\delta$ 141.2, 137.2, 134.1, $128.5,126.5,123.5,121.8,118.8,111.2,107.7,33.7,15.0$.

(E)-1-Methyl-3-(2-nitrovinyl)-2-phenyl-1H-indole (5c). Yellow solid; 96\% yield; mp $156-158{ }^{\circ} \mathrm{C}$; IR (KBr) 750, 806, 982, 1070, 1263, 1304, 1466, $1601 \mathrm{~cm}^{-1}$; HRMS (EI) calcd for $\mathrm{C}_{17} \mathrm{H}_{15} \mathrm{~N}_{2} \mathrm{O}_{2}$ $[\mathrm{M}+\mathrm{H}]^{+}$279.1128, found 279.1124. ${ }^{1} \mathrm{H}$ NMR (600 MHz, DMSO$\left.\mathrm{D}_{6}\right): \delta 8.04(\mathrm{~d}, J=7.8 \mathrm{~Hz}, 1 \mathrm{H}, \mathrm{ArH}), 8.01(\mathrm{~d}, J=13.2 \mathrm{~Hz}, 1 \mathrm{H}, \mathrm{CH})$, 7.91 (d, $J=13.8 \mathrm{~Hz}, 1 \mathrm{H}, \mathrm{CH}), 7.70$ (s, 1H, ArH), 7.69-7.66 (m, 3H, ArH), 7.58-7.57 (m, 2H, ArH), 7.43 (t, J=15.0 Hz, 1H, ArH), 7.36 $(\mathrm{t}, J=15.0 \mathrm{~Hz}, 1 \mathrm{H}, \mathrm{ArH}), 3.69\left(\mathrm{~s}, 3 \mathrm{H}, \mathrm{NCH}_{3}\right) ;{ }^{13} \mathrm{C} \mathrm{NMR}(150 \mathrm{MHz}$, DMSO): $\delta$ 150.1, 138.5, 134.2, 131.7, 131.3, 131.3, 130.5, 129.4, 129.4, 128.9, 124.8, 124.4, 123.3, 121.3, 112.0, 106.5, 32.1.

(E)-3-(2-Nitrovinyl)-1H-indole (5d). Yellow solid; 95\% yield; $\mathrm{mp} 171-173{ }^{\circ} \mathrm{C}$; IR (KBr) 642, 754, 797, 974, 1103, 1319, 1425, 1470, 1516, 1618, 2367, 3402, $3746 \mathrm{~cm}^{-1}$; HRMS (EI) calcd for $\mathrm{C}_{10} \mathrm{H}_{8} \mathrm{NaN}_{2} \mathrm{O}_{2}[\mathrm{M}+\mathrm{Na}]^{+}$211.0478, found 211.0481. ${ }^{1} \mathrm{H}$ NMR (600 MHz, DMSO-D $): \delta 12.25$ (s, $1 \mathrm{H}, \mathrm{NH}), 8.42(\mathrm{~d}, J=13.2 \mathrm{~Hz}, 1 \mathrm{H}$, $\mathrm{CH}), 8.25$ (s, 1H, ArH), 8.02 (d, $J=13.8 \mathrm{~Hz}, 1 \mathrm{H}, \mathrm{CH}), 7.96$ (d, $J=$ $7.8 \mathrm{~Hz}, 1 \mathrm{H}, \mathrm{ArH}), 7.54$ (d, $J=3.9 \mathrm{~Hz}, 1 \mathrm{H}, \mathrm{ArH}), 7.30-7.27$ (m, 1H, ArH), 7.26-7.23 (m, 1H, ArH); ${ }^{13} \mathrm{C}$ NMR (150 MHz, DMSO): $\delta$ 138.2, 136.7, 135.2, 131.6, 125.1, 123.8, 122.4, 120.9, 113.3, 108.7 .

(E)-3-(2-Nitroprop-1-en-1-yl)-1H-indole (5e). Yellow solid; 94\% yield; mp 195-197 ${ }^{\circ} \mathrm{C}$; IR (KBr) 750, 972, 1105, 1224, 1267, 1420, 1630, $3428 \mathrm{~cm}^{-1}$; HRMS (EI) calcd for $\mathrm{C}_{11} \mathrm{H}_{10} \mathrm{NaN}_{2} \mathrm{O}_{2}[\mathrm{M}+$ $\mathrm{Na}]^{+}$225.0634, found 225.0630. ${ }^{1} \mathrm{H}$ NMR (600 MHz, DMSO-D 6 ): $\delta 12.19(\mathrm{~s}, 1 \mathrm{H}, \mathrm{NH}), 8.47$ (s, 1H, ArH), 8.00 (s, 1H, CH), 7.83 (d, $J$ $=7.8 \mathrm{~Hz}, 1 \mathrm{H}, \mathrm{ArH}), 7.52(\mathrm{~d}, J=7.8 \mathrm{~Hz}, 1 \mathrm{H}, \mathrm{ArH}), 7.27-7.24(\mathrm{~m}$, 1H, ArH), 7.21-7.19 (m, 1H, ArH), 2.49 (s, 3H, $\left.\mathrm{CH}_{3}\right) ;{ }^{13} \mathrm{C} \mathrm{NMR}$ (150 MHz, DMSO): $\delta$ 141.5, 136.7, 130.5, 128.0, 127.1, 123.4, 121.5, 118.7, 112.8, 108.7, 15.1 .

\section{General procedure for the synthesis of compounds 4 and 8}

A mixture of (E)-3-(2-nitrovinyl)-indoles (5) (1 mmol), isatins (6) $(1.1 \mathrm{mmol})$ and chiral compounds $7(1.2 \mathrm{mmol})$ in $\mathrm{EtOH} / \mathrm{H}_{2} \mathrm{O}$ $(1: 1 \mathrm{v} / \mathrm{v}, 10 \mathrm{~mL})$ was stirred at reflux for $6 \mathrm{~h}$ at room temperature. The resulting precipitate was collected by filtration and washed with cold $\mathrm{EtOH} / \mathrm{H}_{2} \mathrm{O}(\mathrm{v} / \mathrm{v}=1: 1,3-5 \mathrm{~mL})$ for $2-3$ times to yield pure products ( 4 and 8 ).

$2^{\prime}$-(1-Methyl-1H-indol-3-yl)-1'-nitro-1 $\mathbf{1}^{\prime}, 2^{\prime}, 4 \mathrm{a}^{\prime}, 5^{\prime}, 6^{\prime}, 7^{\prime}, \mathbf{8}^{\prime}, 8 \mathrm{a}^{\prime}, 9^{\prime}, 9 \mathrm{a}^{\prime}$ decahydrospiro[indoline-3, $3^{\prime}$-pyrrolo $[1,2-a]$ indol]-2-one (4a). Yellow solid; 94\% yield; mp 220-222 ${ }^{\circ} \mathrm{C}$; IR (KBr) 745, 1188, 1335, 1472, 1547, 1620, 1721, 2918, $3399 \mathrm{~cm}^{-1}$; HRMS (EI) calcd for $\mathrm{C}_{27} \mathrm{H}_{28} \mathrm{NaN}_{4} \mathrm{O}_{3}[\mathrm{M}+\mathrm{Na}]^{+} 479.2054,479.2057 .{ }^{1} \mathrm{H}$ NMR (400 MHz, DMSO-D $\left.)_{6}\right): \delta .95$ (s, Hz, $\left.1 \mathrm{H}, \mathrm{NH}\right), 7.75(\mathrm{~d}, J=8.0 \mathrm{~Hz}, 1 \mathrm{H}$, $\operatorname{ArH}), 7.41$ (d, $J=8.0 \mathrm{~Hz}, 1 \mathrm{H}, \operatorname{ArH}), 6.98$ (d, $J=4.8 \mathrm{~Hz}, 2 \mathrm{H}, \operatorname{ArH})$, 6.85-6.76 (m, 2H, ArH), $6.67(\mathrm{t}, J=14.8 \mathrm{~Hz}, 2 \mathrm{H}, \mathrm{ArH}), 6.32(\mathrm{t}, J=$ $7.6 \mathrm{~Hz}, 1 \mathrm{H}, \mathrm{ArH}), 6.15(\mathrm{t}, J=21.2 \mathrm{~Hz}, 1 \mathrm{H}, \mathrm{CH}), 4.61(\mathrm{~d}, J=$ $11.6 \mathrm{~Hz}, 1 \mathrm{H}, \mathrm{CH}), 4.48-4.41(\mathrm{~m}, 1 \mathrm{H}, \mathrm{CH}), 3.64(\mathrm{~s}, 1 \mathrm{H}, \mathrm{CH})$, 3.39 (s, 3H, $\mathrm{CH}_{3}$ ), 1.89 (t, $\left.J=10.4 \mathrm{~Hz}, 1 \mathrm{H}, \mathrm{CH}\right), 1.56-1.49$ (m, $\left.1 \mathrm{H}, \mathrm{CH}_{2}\right), 1.33-1.27\left(\mathrm{~m}, 3 \mathrm{H}, \mathrm{CH}_{2}\right), 1.05-0.99\left(\mathrm{~m}, 2 \mathrm{H}, \mathrm{CH}_{2}\right), 0.95-$ $0.81\left(\mathrm{~m}, 2 \mathrm{H}, \mathrm{CH}_{2}\right), 0.76-0.79\left(\mathrm{~m}, 1 \mathrm{H}, \mathrm{CH}_{2}\right), 0.29-0.32(\mathrm{~m}, 1 \mathrm{H}$, $\left.\mathrm{CH}_{2}\right) ;{ }^{13} \mathrm{C}$ NMR (100 MHz, DMSO): $\delta$ 179.5, 143.6, 136.4, 130.0, 128.2, 127.9, 127.3, 125.2, 121.6, 121.6, 119.3, 119.1, 109.9, 109.8, 107.1, 91.7, 73.8, 63.2, 59.0, 43.0, 38.3, 34.2, 32.9, 29.2, 27.3, 24.8, 19.6 .

5-Methyl-2'-(1-methyl-1H-indol-3-yl)-1'-nitro-1' $2^{\prime}, 4 a^{\prime}, 5^{\prime}, 6^{\prime}, 7^{\prime}$, $8^{\prime}, 8 a^{\prime}, 9^{\prime}, 9 a^{\prime}$-decahydrospiro[indoline-3, $3^{\prime}$-pyrrolo[1,2-a $]$ indol]-2one (4b). Yellow solid; 92\% yield; mp 221-223 ${ }^{\circ} \mathrm{C}$; IR ( $\left.\mathrm{KBr}\right) 741$, 812, 1177, 1211, 1333, 1491, 1537, 1707, 2934, $3482 \mathrm{~cm}^{-1}$; HRMS (EI) calcd for $\mathrm{C}_{28} \mathrm{H}_{30} \mathrm{NaN}_{4} \mathrm{O}_{3}[\mathrm{M}+\mathrm{Na}]^{+}$493.2210, found 493.2215. ${ }^{1} \mathrm{H}$ NMR (400 MHz, DMSO-D $): \delta 10.06$ (s, 1H, NH), $7.84(\mathrm{~s}, 1 \mathrm{H}, \operatorname{ArH}), 7.63$ (d, $J=8.0 \mathrm{~Hz}, 1 \mathrm{H}, \operatorname{ArH}), 7.23(\mathrm{~d}, J=$ $6.8 \mathrm{~Hz}, 2 \mathrm{H}, \mathrm{ArH}), 7.03(\mathrm{t}, J=14.8 \mathrm{~Hz}, 1 \mathrm{H}, \mathrm{ArH}), 6.93-6.86(\mathrm{~m}, 2 \mathrm{H}$, ArH), 6.42 (t, $J=13.2 \mathrm{~Hz}, 1 \mathrm{H}, \mathrm{ArH}), 6.37$ (d, $J=9.6 \mathrm{~Hz}, 1 \mathrm{H}, \mathrm{CH})$, 4.82-4.79 (m, 1H, CH), 4.71-4.65 (m, 1H, CH), $3.88\left(\mathrm{~s}, 1 \mathrm{H}, \mathrm{CH}_{2}\right)$, $3.64\left(\mathrm{~s}, 3 \mathrm{H}, \mathrm{NCH}_{3}\right), 2.30\left(\mathrm{~s}, 3 \mathrm{H}, \mathrm{CH}_{3}\right), 2.24-2.13\left(\mathrm{~m}, 1 \mathrm{H}, \mathrm{CH}_{2}\right)$, 1.79-1.73 (m, 1H, $\left.\mathrm{CH}_{2}\right), 1.57-1.53\left(\mathrm{~m}, 3 \mathrm{H}, \mathrm{CH}_{2}\right), 1.29-1.20(\mathrm{~m}$, $\left.2 \mathrm{H}, \mathrm{CH}_{2}\right), 1.17-1.06\left(\mathrm{~m}, 2 \mathrm{H}, \mathrm{CH}_{2}\right), 0.89-0.94\left(\mathrm{~m}, 1 \mathrm{H}, \mathrm{CH}_{2}\right), 0.56-$ $0.61\left(\mathrm{~m}, 1 \mathrm{H}, \mathrm{CH}_{2}\right) ;{ }^{13} \mathrm{C} \mathrm{NMR}$ (100 MHz, DMSO): $\delta$ 179.4, 141.1, $136.4,130.5$, 130.0, 128.1, 128.0, 127.9, 125.2, 121.6, 119.3, 118.9, 110.0, 109.4, 107.1, 91.5, 73.8, 63.2, 59.0, 43.2, 38.3, 34.2, $32.9,29.2,27.3,24.8,21.1,19.6$.

5-Methoxy-2'-(1-methyl-1H-indol-3-yl)-1'-nitro-1', $2^{\prime}, 4 \mathrm{a}^{\prime}, 5^{\prime}, 6^{\prime}$, $7^{\prime}, \mathbf{8}^{\prime}, 8 \mathrm{a}^{\prime}, \mathbf{9}^{\prime}, \mathbf{9 a}^{\prime}$-decahydrospiro[indoline-3, $\mathbf{3}^{\prime}$-pyrrolo[1,2-a]indol]-2one (4c). Yellow solid; 91\% yield; mp 219-221 ${ }^{\circ} \mathrm{C}$; IR (KBr) 745, 814, 1032, 1206, 1333, 1489, 1543, 1714, 2926, 3234, $3406 \mathrm{~cm}^{-1}$; HRMS (EI) calcd for $\mathrm{C}_{28} \mathrm{H}_{31} \mathrm{~N}_{4} \mathrm{O}_{4}[\mathrm{M}+\mathrm{H}]^{+}$487.2340, found 487.2336. ${ }^{1} \mathrm{H}$ NMR (600 MHz, DMSO-D 6$): \delta 9.99(\mathrm{~s}, 1 \mathrm{H}, \mathrm{NH})$, 7.78-7.74 (m, 1H, ArH), 7.25 (s, 1H, ArH), $7.22(\mathrm{~d}, J=9.0 \mathrm{~Hz}, 1 \mathrm{H}$, ArH), 7.05-7.02 (m, 1H, ArH), 6.94 (t, $J=7.8 \mathrm{~Hz}, 1 \mathrm{H} \mathrm{ArH}), 6.92$ (d, $J=0.6 \mathrm{~Hz}, 1 \mathrm{H}, \mathrm{ArH}), 6.65-6.63(\mathrm{~m}, 1 \mathrm{H}, \mathrm{ArH}), 6.46$ (d, $J=$ $8.4 \mathrm{~Hz}, 1 \mathrm{H}, \mathrm{ArH}), 6.41-6.37(\mathrm{~m}, 1 \mathrm{H}, \mathrm{CH}), 4.89(\mathrm{~d}, J=11.4 \mathrm{~Hz}, 1 \mathrm{H}$, $\mathrm{CH}), 4.68-4.66(\mathrm{~m}, 1 \mathrm{H}, \mathrm{CH}), 3.94(\mathrm{~d}, J=1.8 \mathrm{~Hz}, 1 \mathrm{H}, \mathrm{CH}), 3.75$ (s, $3 \mathrm{H}, \mathrm{NCH}_{3}$ ), 3.65 (s, 3H, $\mathrm{OCH}_{3}$ ), 2.15 (d, $\left.J=6.0 \mathrm{~Hz}, 1 \mathrm{H}, \mathrm{CH}\right), 1.79$ $\left(\mathrm{t}, J=10.2 \mathrm{~Hz}, 1 \mathrm{H}, \mathrm{CH}_{2}\right), 1.55-1.52\left(\mathrm{~m}, 3 \mathrm{H}, \mathrm{CH}_{2}\right), 1.26(\mathrm{t}, J=$ 13.2 Hz, $\left.1 \mathrm{H}, \mathrm{CH}_{2}\right), 1.24-1.17\left(\mathrm{~m}, 1 \mathrm{H}, \mathrm{CH}_{2}\right), 1.07-1.01(\mathrm{~m}, 2 \mathrm{H}$, $\mathrm{CH}_{2}$ ), 0.95 (d, $\left.J=3.0 \mathrm{~Hz}, 1 \mathrm{H}, \mathrm{CH}_{2}\right), 0.55(\mathrm{~d}, J=13.8 \mathrm{~Hz}, 1 \mathrm{H}$, 
$\mathrm{CH}_{2}$ ); ${ }^{13} \mathrm{C}$ NMR (150 MHz, DMSO): $\delta$ 179.5, 155.1, 136.9, 136.4, $128.3,127.8,126.4,121.6,119.4,119.0,115.5,114.2,110.0$, 109.9, 107.2, 91.7, 74.2, 63.2, 58.8, 56.4, 42.9, 38.3, 34.2, 32.9, 29.2, 27.3, 24.8, 19.7 .

5-Fluoro-2' -(1-methyl-1H-indol-3-yl)-1' ${ }^{\prime}$-nitro-1' $\mathbf{2}^{\prime}, \mathbf{4 a}^{\prime}, 5^{\prime}, 6^{\prime}, 7^{\prime}$, $8^{\prime}, 8 a^{\prime}, 9^{\prime}, 9 a^{\prime}$-decahydrospiro[indoline-3,3' -pyrrolo[1,2-a]indol]-2one (4d). Yellow solid; 95\% yield; mp 243-245 ${ }^{\circ} \mathrm{C}$; IR (KBr) 743, 820, 1177, 1329, 1375, 1489, 1547, 1720, 2938, 3410, $3424 \mathrm{~cm}^{-1}$; HRMS (EI) calcd for $\mathrm{C}_{27} \mathrm{H}_{27} \mathrm{NaFN}_{4} \mathrm{O}_{3}[\mathrm{M}+\mathrm{Na}]^{+} 497.1959$, found 497.1957. ${ }^{1} \mathrm{H}$ NMR (600 MHz, DMSO-D $): \delta 10.21$ (s, $1 \mathrm{H}, \mathrm{NH}$ ), 8.09 (d, $J=7.8 \mathrm{~Hz}, 1 \mathrm{H}, \operatorname{ArH}), 7.74(\mathrm{~d}, J=7.8 \mathrm{~Hz}, 1 \mathrm{H}, \operatorname{ArH}), 7.24$ (d, $J=9.0 \mathrm{~Hz}, 2 \mathrm{H}, \mathrm{ArH}), 7.04$ (d, $J=7.2 \mathrm{~Hz}, 1 \mathrm{H}, \mathrm{ArH}), 6.96-6.92$ (m, 2H, ArH), 6.55 (s, 1H, ArH), 6.40 (t, J=20.4 Hz, 1H, CH), 4.91 (d, $J=11.4 \mathrm{~Hz}, 1 \mathrm{H}, \mathrm{CH}), 4.71(\mathrm{~d}, J=6.6 \mathrm{~Hz}, 1 \mathrm{H}, \mathrm{CH}), 3.93(\mathrm{~s}, 1 \mathrm{H}$, $\mathrm{CH}), 3.64\left(\mathrm{~s}, 3 \mathrm{H}, \mathrm{NCH}_{3}\right), 2.16(\mathrm{~d}, J=3.6 \mathrm{~Hz}, 1 \mathrm{H}, \mathrm{CH}), 1.81(\mathrm{~d}, J=$ $\left.4.8 \mathrm{~Hz}, 1 \mathrm{H}, \mathrm{CH}_{2}\right), 1.55\left(\mathrm{~s}, 3 \mathrm{H}, \mathrm{CH}_{3}\right), 1.27-1.19\left(\mathrm{~m}, 2 \mathrm{H}, \mathrm{CH}_{2}\right)$, 1.09-0.99 (m, 3H, $\left.\mathrm{CH}_{2}\right), 0.55$ (d, $\left.J=12.6 \mathrm{~Hz}, 1 \mathrm{H}, \mathrm{CH}_{2}\right) ;{ }^{13} \mathrm{C} \mathrm{NMR}$ (150 MHz, DMSO): $\delta$ 179.5, 159.0, 157.5, 140.0, 136.4, 128.2, $127.9,121.7,119.3,119.1,116.3,116.2,115.5,110.4,110.4$, 106.9, 91.3, 74.1, 63.3, 58.8, 43.2, 38.3, 34.2, 32.9, 29.2, 27.3, 24.8, 19.6 .

6-Chloro-2' -(1-methyl-1H-indol-3-yl)-1'-nitro-1' $\mathbf{2}^{\prime}, \mathbf{4 a}^{\prime}, 5^{\prime}, 6^{\prime}, 7^{\prime}$, $8^{\prime}, 8 a^{\prime}, 9^{\prime}, 9 a^{\prime}$-decahydrospiro[indoline-3,3' -pyrrolo[1,2-a]indol]-2one (4e). Yellow solid; 95\% yield; mp 182-184 ${ }^{\circ} \mathrm{C}$; IR (KBr) 739, 1072, 1126, 1333, 1449, 1543, 1609, 1707, 2936, 3248, 3416, 3503, $3624 \mathrm{~cm}^{-1}$; HRMS (EI) calcd for $\mathrm{C}_{27} \mathrm{H}_{27} \mathrm{NaClN}_{4} \mathrm{O}_{3}[\mathrm{M}+$ $\mathrm{Na}]^{+}$513.1664, found 513.1668. ${ }^{1} \mathrm{H}$ NMR (600 MHz, DMSO-D 6 ): $\delta 10.36(\mathrm{~s}, 1 \mathrm{H}, \mathrm{NH}), 8.08\left(\mathrm{~d}, J=7.8 \mathrm{~Hz}, 1 \mathrm{H}, \mathrm{CH}_{2}\right), 7.66(\mathrm{~d}, J=$ $\left.7.2 \mathrm{~Hz}, 1 \mathrm{H}, \mathrm{CH}_{2}\right), 7.25\left(\mathrm{~d}, J=12.0 \mathrm{~Hz}, 2 \mathrm{H}, \mathrm{CH}_{2}\right), 7.06(\mathrm{t}, J=$ $\left.14.4 \mathrm{~Hz}, 1 \mathrm{H}, \mathrm{CH}_{2}\right), 6.98\left(\mathrm{~d}, J=7.2 \mathrm{~Hz}, 1 \mathrm{H}, \mathrm{CH}_{2}\right), 6.93(\mathrm{t}$, $\left.J=14.4 \mathrm{~Hz}, 1 \mathrm{H}, \mathrm{CH}_{2}\right), 6.59\left(\mathrm{~s}, 1 \mathrm{H}, \mathrm{CH}_{2}\right), 6.38(\mathrm{t}, J=21.0 \mathrm{~Hz}, 1 \mathrm{H}$, $\mathrm{CH}), 4.88(\mathrm{~d}, J=11.4 \mathrm{~Hz}, 1 \mathrm{H}, \mathrm{CH}), 4.69$ (d, $J=7.2 \mathrm{~Hz}, 1 \mathrm{H}, \mathrm{CH})$, $3.87(\mathrm{~s}, 1 \mathrm{H}, \mathrm{CH}), 3.65\left(\mathrm{~s}, 1 \mathrm{H}, \mathrm{NCH}_{3}\right), 2.16(\mathrm{~d}, J=5.4 \mathrm{~Hz}, 1 \mathrm{H}, \mathrm{CH})$, 1.80-1.76 (m, 1H, $\left.\mathrm{CH}_{2}\right), 1.57-1.52\left(\mathrm{~m}, 3 \mathrm{H}, \mathrm{CH}_{2}\right), 1.28-1.15(\mathrm{~m}$, $\left.2 \mathrm{H}, \mathrm{CH}_{2}\right), 1.09-0.94\left(\mathrm{~m}, 3 \mathrm{H}, \mathrm{CH}_{2}\right), 0.57-0.54\left(\mathrm{~m}, 1 \mathrm{H}, \mathrm{CH}_{2}\right) ;{ }^{13} \mathrm{C}$ NMR (150 MHz, DMSO): $\delta$ 179.4, 145.1, 136.4, 134.4, 128.9, $128.1,128.0,124.2$, 121.7, 121.3, 119.2, 119.1, 110.0, 110.0, 106.7, 91.3, 73.5, 63.3, 59.0, 49.1, 38.3, 34.2, 32.9, 29.3, 27.2, 24.7, 19.6 .

6-Bromo-2' -(1-methyl-1H-indol-3-yl)-1'-nitro-1' $, 2^{\prime}, 4 a^{\prime}, 5^{\prime}, 6^{\prime}, 7^{\prime}$, $8^{\prime}, 8 a^{\prime}, 9^{\prime}, 9 a^{\prime}$-decahydrospiro[indoline-3,3'-pyrrolo[1,2-a]indol]-2one (4f). Yellow solid; 96\% yield; mp $223-225^{\circ} \mathrm{C}$; IR (KBr) 739, 1067, 1128, 1333, 1447, 1481, 1543, 1607, 1107, 2934, 3258, 3416, 3501, $3622 \mathrm{~cm}^{-1}$; HRMS (EI) calcd for $\mathrm{C}_{27} \mathrm{H}_{27} \mathrm{NaBrN}_{4} \mathrm{O}_{3}[\mathrm{M}$ $+\mathrm{Na}]^{+}$557.1159, found 557.1164. ${ }^{1} \mathrm{H}$ NMR (600 MHz, DMSO-D 6 ): $\delta 10.30(\mathrm{~s}, 1 \mathrm{H}, \mathrm{NH}), 8.01$ (d, $J=6.0 \mathrm{~Hz}, 1 \mathrm{H}, \operatorname{ArH}), 7.66$ (d, $J=$ $7.8 \mathrm{~Hz}, 1 \mathrm{H}, \operatorname{ArH}), 7.25$ (t, $J=19.2 \mathrm{~Hz}, 2 \mathrm{H}, \operatorname{ArH}), 7.12(\mathrm{t}, J=$ $8.4 \mathrm{~Hz}, 1 \mathrm{H}, \operatorname{ArH}), 7.05(\mathrm{t}, J=15.0 \mathrm{~Hz}, 1 \mathrm{H}, \operatorname{ArH}), 6.93(\mathrm{t}$, $J=14.4 \mathrm{~Hz}, 1 \mathrm{H}, \mathrm{ArH}), 6.72(\mathrm{~d}, J=1.8 \mathrm{~Hz}, 1 \mathrm{H}, \mathrm{ArH}), 6.39-6.35(\mathrm{~m}$, $1 \mathrm{H}, \mathrm{CH}), 4.93$ (d, $J=10.8 \mathrm{~Hz}, 1 \mathrm{H}, \mathrm{CH}), 4.68(\mathrm{~d}, J=6.6 \mathrm{~Hz}, 1 \mathrm{H}$, $\mathrm{CH}), 3.92$ (s, $1 \mathrm{H}, \mathrm{CH}), 3.65\left(\mathrm{~s}, 3 \mathrm{H}, \mathrm{CH}_{3}\right), 3.19$ (d, $J=4.8 \mathrm{~Hz}, 1 \mathrm{H}$, $\mathrm{CH})$, 2.16-2.14 (m, 1H, $\left.\mathrm{CH}_{2}\right), 1.79-1.76\left(\mathrm{~m}, 1 \mathrm{H}, \mathrm{CH}_{2}\right), 1.57-1.52$ (m, 3H, $\mathrm{CH}_{2}$ ), 1.25-1.16 (m, 2H, $\left.\mathrm{CH}_{2}\right), 1.10-0.98\left(\mathrm{~m}, 3 \mathrm{H}, \mathrm{CH}_{2}\right)$; ${ }^{13} \mathrm{C}$ NMR (150 MHz, DMSO): $\delta$ 179.3, 145.3, 136.4, 129.2, 128.1, 128.0, 124.6, 124.2, 122.9, 121.7, 119.2, 112.7, 110.0, 106.7, 91.4, 73.6, 63.3, 59.0, 49.1, 43.1, 38.3, 34.2, 32.9, 29.3, 27.2, 24.7, 19.6.
5-Bromo-2'-(1-methyl-1H-indol-3-yl)-1'-nitro-1 ${ }^{\prime}, 2^{\prime}, 4 a^{\prime}, 5^{\prime}, 6^{\prime}, 7^{\prime}$, $8^{\prime}, 8 a^{\prime}, 9^{\prime}, 9 a^{\prime \prime}$-decahydrospiro[indoline-3, $3^{\prime}$-pyrrolo[1,2-a]indol]2-one (4g). Yellow solid; 94\% yield; mp 223-225 ${ }^{\circ} \mathrm{C}$; IR (KBr) 739, 816, 1186, 1331, 1477, 1545, 1616, 1717, 2363, 2930, 3395, 3426, $3624 \mathrm{~cm}^{-1}$; HRMS (EI) calcd for $\mathrm{C}_{27} \mathrm{H}_{27} \mathrm{NaBrN}_{4} \mathrm{O}_{3}[\mathrm{M}+\mathrm{Na}]^{+}$ 557.1159, found 557.1157. ${ }^{1} \mathrm{H}$ NMR (600 MHz, DMSO-D ${ }_{6}$ ): $\delta 10.30$ (s, 1H, NH), 8.37 (s, 1H, ArH), 7.69 (d, $J=7.2 \mathrm{~Hz}, 1 \mathrm{H}$, ArH), 7.24 (d, J=7.2 Hz, 3H, ArH), 7.05 (t, J=13.2 Hz, 1H, ArH), $6.94(\mathrm{~d}, J=6.6 \mathrm{~Hz}, 1 \mathrm{H}, \operatorname{ArH}), 6.51(\mathrm{~d}, J=7.8 \mathrm{~Hz}, 1 \mathrm{H}, \operatorname{ArH}), 6.38(\mathrm{t}$, $J=20.4 \mathrm{~Hz}, 1 \mathrm{H}, \mathrm{CH}), 4.93(\mathrm{~d}, J=10.8 \mathrm{~Hz}, 1 \mathrm{H}, \mathrm{CH}), 4.68$ (d, $J=$ $6.6 \mathrm{~Hz}, 1 \mathrm{H}, \mathrm{CH}), 3.92(\mathrm{~s}, 1 \mathrm{H}, \mathrm{CH}), 3.65\left(\mathrm{~s}, 3 \mathrm{H}, \mathrm{CH}_{3}\right), 2.16(\mathrm{~d}, J=$ $4.2 \mathrm{~Hz}, 1 \mathrm{H}, \mathrm{CH}), 1.83$ (d, $\left.J=4.8 \mathrm{~Hz}, 1 \mathrm{H}, \mathrm{CH}_{2}\right), 1.54(\mathrm{~d}, J=6.0 \mathrm{~Hz}$, $\left.3 \mathrm{H}, \mathrm{CH}_{2}\right), 1.25-1.16\left(\mathrm{~m}, 2 \mathrm{H}, \mathrm{CH}_{2}\right), 1.10-0.98\left(\mathrm{~m}, 3 \mathrm{H}, \mathrm{CH}_{2}\right), 0.56$ (d, $\left.J=13.2 \mathrm{~Hz}, 1 \mathrm{H}, \mathrm{CH}_{2}\right) ;{ }^{13} \mathrm{C}$ NMR (150 MHz, DMSO): $\delta$ 179.1, 142.9, 136.5, 132.6, 130.4, 128.1, 127.8, 127.7, 121.7, 119.4, 119.0, 113.7, 111.6, 110.0, 106.8, 91.0, 74.0, 63.4, 58.8, 43.2, 38.3, $34.2,32.9,29.3,27.2,24.8,19.6$.

5-Methoxy-1'-methyl-2' -(1-methyl-1H-indol-3-yl)-1'-nitro-

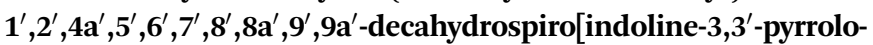
[1,2-a]indol]-2-one (4h). Yellow solid; 87\% yield; mp $215-217^{\circ} \mathrm{C}$; IR (KBr) 741, 1042, 1120, 1489, 1543, 1732, 2365, 2930, $3304 \mathrm{~cm}^{-1}$; HRMS (EI) calcd for $\mathrm{C}_{29} \mathrm{H}_{33} \mathrm{~N}_{4} \mathrm{O}_{4}[\mathrm{M}+\mathrm{H}]^{+}$501.2496, found 501.2499. ${ }^{1} \mathrm{H}$ NMR (500 MHz, DMSO-D $): \delta 10.30(\mathrm{~s}, 1 \mathrm{H}$, $\mathrm{NH}), 7.84$ (d, $J=7.5 \mathrm{~Hz}, 1 \mathrm{H}, \mathrm{ArH}), 7.53$ (s, 1H, ArH), 7.39 (d, $J=$ $8.5 \mathrm{~Hz}, 1 \mathrm{H}, \mathrm{ArH}), 7.17-7.11$ (m, 1H, ArH), 7.09 (d, J=7.5 Hz, 2H, ArH), 6.89-6.86 (m, 1H, ArH), 6.74 (d, $J=8.5 \mathrm{~Hz}, 1 \mathrm{H}, \mathrm{ArH}), 4.52$ $(\mathrm{d}, J=11.0 \mathrm{~Hz}, 1 \mathrm{H}, \mathrm{CH}), 4.26-4.25(\mathrm{~m}, 1 \mathrm{H}, \mathrm{CH}), 3.80(\mathrm{~s}, 3 \mathrm{H}$, $\mathrm{NCH}_{3}$ ), 3.78 (s, 3H, $\mathrm{OCH}_{3}$ ), 3.17 (d, $\left.J=3.5 \mathrm{~Hz}, 1 \mathrm{H}, \mathrm{CH}\right), 2.09-$ $2.06\left(\mathrm{~m}, 1 \mathrm{H}, \mathrm{CH}_{2}\right), 1.89\left(\mathrm{~s}, 3 \mathrm{H}, \mathrm{CH}_{3}\right), 1.76-1.75\left(\mathrm{~m}, 1 \mathrm{H}, \mathrm{CH}_{2}\right)$, 1.56-1.52 (m, 2H, $\left.\mathrm{CH}_{2}\right), 1.46-1.36\left(\mathrm{~m}, 3 \mathrm{H}, \mathrm{CH}_{2}\right), 1.19-1.12(\mathrm{~m}$, $2 \mathrm{H}, \mathrm{CH}_{2}$ ), 1.03-0.96 (m, 2H, $\left.\mathrm{CH}_{2}\right) ;{ }^{13} \mathrm{C} \mathrm{NMR}$ (125 MHz, DMSO): $\delta 177.5,154.4,136.8,136.3,130.0,128.6,125.4,121.5,120.1$, 119.3, 115.1, 114.3, 110.6, 110.1, 106.8, 101.4, 74.7, 66.7, 58.0, 56.1, 48.6, 40.4, 37.4, 33.0, 27.8, 27.6, 24.7, 20.2, 19.7.

5-Fluoro-1' -methyl-2' -(1-methyl-1H-indol-3-yl)-1' -nitro-1 $^{\prime}, \mathbf{2}^{\prime}$, $4 a^{\prime}, 5^{\prime}, 6^{\prime}, 7^{\prime}, 8^{\prime}, 8 a^{\prime}, 9^{\prime}, 9 a^{\prime}$-decahydrospiro[indoline-3, $3^{\prime}$-pyrrolo[1,2a]indol]-2-one (4i). Yellow solid; 92\% yield; mp $170-172{ }^{\circ} \mathrm{C}$; IR (KBr) 741, 818, 1182, 1333, 1487, 1537, 1717, 2930, 3393, $3426 \mathrm{~cm}^{-1}$; HRMS (EI) calcd for $\mathrm{C}_{28} \mathrm{H}_{28} \mathrm{NaFN}_{4} \mathrm{O}_{3}[\mathrm{M}+\mathrm{Na}]^{+}$ 511.2116, found 511.2120. ${ }^{1} \mathrm{H}$ NMR (500 MHz, DMSO-D 6 ): $\delta 10.31(\mathrm{~s}, 1 \mathrm{H}, \mathrm{NH}), 7.76-7.73(\mathrm{~m}, 1 \mathrm{H}, \mathrm{ArH}), 7.67$ (d, $J=8.0 \mathrm{~Hz}$, $1 \mathrm{H}, \operatorname{ArH}), 7.27$ (d, J = 8.0 Hz, 1H, ArH), 7.11 (s, 1H, ArH), 7.087.01 (m, 1H, ArH), 6.99-6.91 (m, 1H, ArH), 6.90-6.88 (m, 1H, ArH), 6.60-6.57 (m, 1H, ArH), 4.44-4.41 (m, 1H, CH), 3.74 (d, $J=$ $3.5 \mathrm{~Hz}, 1 \mathrm{H}, \mathrm{CH}), 3.69$ (s, 3H, $\mathrm{NCH}_{3}$ ), 2.13-2.09 (m, 1H, CH), 2.08 (s, 3H, $\mathrm{CH}_{3}$ ), 1.73-1.69 (m, $\left.1 \mathrm{H}, \mathrm{CH}_{2}\right), 1.59-1.55\left(\mathrm{~m}, 1 \mathrm{H}, \mathrm{CH}_{2}\right)$, 1.52-1.50 (m, 2H, $\left.\mathrm{CH}_{2}\right), 1.27-1.24\left(\mathrm{~m}, 1 \mathrm{H}, \mathrm{CH}_{2}\right), 1.19-1.18(\mathrm{~m}$, $\left.1 \mathrm{H}, \mathrm{CH}_{2}\right), 1.07-1.02\left(\mathrm{~m}, 2 \mathrm{H}, \mathrm{CH}_{2}\right), 1.00-0.91\left(\mathrm{~m}, 1 \mathrm{H}, \mathrm{CH}_{2}\right), 0.55-$ $0.52\left(\mathrm{~m}, 1 \mathrm{H}, \mathrm{CH}_{2}\right) ;{ }^{13} \mathrm{C} \mathrm{NMR}$ (125 MHz, DMSO): $\delta$ 180.3, 159.1, 157.2 , 139.6, 136.0, 129.2, 128.9, 128.4, 121.7, 119.4, 118.7, 116.4, 114.2, 110.4, 110.4, 104.9, 99.8, 74.4, 73.9, 58.1, 50.3, 38.9, $36.3,33.0,28.7,27.5,25.9,24.5,19.4$.

5-Chloro-1' -methyl-2' -(1-methyl-1H-indol-3-yl)-1' -nitro-1' $\mathbf{2}^{\prime}$, $4 a^{\prime}, 5^{\prime}, 6^{\prime}, 7^{\prime}, 8^{\prime}, 8 a^{\prime}, 9^{\prime}, 9 a^{\prime}$-decahydrospiro[indoline-3, $3^{\prime}$-pyrrolo[1,2a]indol]-2-one (4j). Yellow solid; 92\% yield; mp $183-185{ }^{\circ} \mathrm{C}$; IR (KBr) 743, 820, 1194, 1474, 1543, 1614, 1738, 2859, 2928, 
$3308 \mathrm{~cm}^{-1}$; HRMS (EI) calcd for $\mathrm{C}_{28} \mathrm{H}_{29} \mathrm{NaClN}_{4} \mathrm{O}_{3}[\mathrm{M}+\mathrm{Na}]^{+}$ 527.1820, found 527.1825. ${ }^{1} \mathrm{H}$ NMR (500 MHz, DMSO-D 6 ): $\delta 10.64(\mathrm{~s}, 1 \mathrm{H}, \mathrm{NH}), 7.88$ (d, $J=7.5 \mathrm{~Hz}, 1 \mathrm{H}, \operatorname{ArH}), 7.73(\mathrm{~s}, 1 \mathrm{H}$, $\operatorname{ArH}), 7.53$ (s, 1H, ArH), 7.47 (d, J=8.0 Hz, 1H, ArH), 7.40 (d, $J=$ $8.0 \mathrm{~Hz}, 1 \mathrm{H}, \operatorname{ArH}), 7.16(\mathrm{t}, J=14.5 \mathrm{~Hz}, 1 \mathrm{H}, \operatorname{ArH}), 7.10(\mathrm{t}$, $J=14.5 \mathrm{~Hz}, 1 \mathrm{H}, \mathrm{ArH}), 6.80(\mathrm{~d}, J=8.0 \mathrm{~Hz}, 1 \mathrm{H}, \mathrm{ArH}), 4.52(\mathrm{~d}, J=$ $10.5 \mathrm{~Hz}, 1 \mathrm{H}, \mathrm{CH}), 4.32-4.27(\mathrm{~m}, 1 \mathrm{H}, \mathrm{CH}), 3.78\left(\mathrm{~s}, 3 \mathrm{H}, \mathrm{NCH}_{3}\right)$, $3.16(\mathrm{~s}, 1 \mathrm{H}, \mathrm{CH}), 2.08$ (d, $J=5.0 \mathrm{~Hz}, 1 \mathrm{H}, \mathrm{CH}), 1.90$ (d, $J=15.5 \mathrm{~Hz}$, $\left.3 \mathrm{H}, \mathrm{CH}_{3}\right), 1.54-1.51\left(\mathrm{~m}, 3 \mathrm{H}, \mathrm{CH}_{2}\right), 1.42-1.35\left(\mathrm{~m}, 3 \mathrm{H}, \mathrm{CH}_{2}\right), 1.15-$ $1.13\left(\mathrm{~m}, 1 \mathrm{H}, \mathrm{CH}_{2}\right), 1.03-0.92\left(\mathrm{~m}, 3 \mathrm{H}, \mathrm{CH}_{2}\right) ;{ }^{13} \mathrm{C} \mathrm{NMR}(125 \mathrm{MHz}$, DMSO): $\delta$ 177.3, 142.3, 136.8, 133.4, 130.2, 129.7, 128.5, 126.6, $121.6,120.3$, 119.3, 113.3, 112.4, 110.1, 106.6, 101.8, 74.5, 66.5, 58.2, 48.8, 41.0, 37.1, 33.0, 27.8, 27.6, 24.7, 20.3, 19.6.

5-Fluoro-2'-(1-methyl-2-phenyl-1H-indol-3-yl)-1' ${ }^{\prime}$-nitro-1' $\mathbf{2}^{\prime}$, $4 a^{\prime}, 5^{\prime}, 6^{\prime}, 7^{\prime}, 8^{\prime}, 8 a^{\prime}, 9^{\prime}, 9 a^{\prime}$-decahydrospiro[indoline-3, $3^{\prime}$-pyrrolo[1,2$a$ ]indol]-2-one (4k). Yellow solid; 91\% yield; mp $210-213{ }^{\circ} \mathrm{C}$; IR (KBr) 743, 1182, 1364, 1487, 1551, 1724, 1734, 2857, 2928, $3345 \mathrm{~cm}^{-1}$; HRMS (EI) calcd for $\mathrm{C}_{33} \mathrm{H}_{31} \mathrm{NaFN}_{4} \mathrm{O}_{3}[\mathrm{M}+\mathrm{Na}]^{+}$ 573.2272, found 573.2269. ${ }^{1} \mathrm{H}$ NMR (500 MHz, DMSO-D 6 ): $\delta 10.67$ (s, 1H, NH), 7.88 (d, $J=6.5 \mathrm{~Hz}, 1 \mathrm{H}, \operatorname{ArH}), 7.63$ (d, $J=$ $7.5 \mathrm{~Hz}, 3 \mathrm{H}, \mathrm{ArH}), 7.53$ (d, $J=8.0 \mathrm{~Hz}, 3 \mathrm{H}, \mathrm{ArH}), 7.28-7.25(\mathrm{~m}, 1 \mathrm{H}$, ArH), 7.22-7.20 (m, 1H, ArH), 7.15-7.11 (m, 1H, ArH), 6.86-6.81 (m, 2H, ArH), 5.98 (d, J = $10.0 \mathrm{~Hz}, 1 \mathrm{H}, \mathrm{CH}), 4.33(\mathrm{~s}, 1 \mathrm{H}, \mathrm{CH})$, 4.05-4.00 (m, 1H, CH), $3.51\left(\mathrm{~s}, 3 \mathrm{H}, \mathrm{NCH}_{3}\right), 3.10(\mathrm{~s}, 1 \mathrm{H}, \mathrm{CH})$, 2.04-2.02 (m, 1H, CH), 1.65-1.40 (m, 4H, $\left.\mathrm{CH}_{2}\right), 1.31-1.12(\mathrm{~m}$, $3 \mathrm{H}, \mathrm{CH}_{2}$ ), 0.98-0.85 (m, 3H, $\left.\mathrm{CH}_{2}\right) ;{ }^{13} \mathrm{C} \mathrm{NMR}$ (125 MHz, DMSO): $\delta$ 178.6, 158.6, 156.7, 139.6, 137.3, 131.3, 131.1, 129.5, 129.0, $123.5,123.5,122.2,120.2,118.9,117.6,117.4,114.4,114.3$, $111.8,111.8,111.1,105.9,94.4,72.0,66.6,57.9,46.4,41.0,37.8$, 31.0, 27.9, 27.4, 24.6, 19.6 .

5-Bromo-2' -(1-methyl-2-phenyl-1H-indol-3-yl)-1' -nitro-1' $\mathbf{2}^{\prime}$, $4 a^{\prime}, 5^{\prime}, 6^{\prime}, 7^{\prime}, 8^{\prime}, 8 a^{\prime}, 9^{\prime}, 9 a^{\prime}$-decahydrospiro[indoline-3, $3^{\prime}$-pyrrolo[1,2a]indol]-2-one (4l). Yellow solid; 92\% yield; mp $185-188^{\circ} \mathrm{C}$; IR (KBr) 702, 743, 818, 1192, 1366, 1470, 1551, 1612, 1734, 2855, 2928, 3252, $3366 \mathrm{~cm}^{-1}$; HRMS (EI) calcd for $\mathrm{C}_{33} \mathrm{H}_{31} \mathrm{NaBrN}_{4} \mathrm{O}_{3}[\mathrm{M}$ $+\mathrm{Na}]^{+}$633.1472, found 633.1474. ${ }^{1} \mathrm{H}$ NMR (500 MHz, DMSO-D 6 ): $\delta 10.80(\mathrm{~s}, 1 \mathrm{H}, \mathrm{NH}), 7.81(\mathrm{t}, J=14.5 \mathrm{~Hz}, 1 \mathrm{H}, \mathrm{ArH}), 7.62-7.56(\mathrm{~m}$, $3 \mathrm{H}, \mathrm{ArH}$ ), 7.53-7.50 (m, 2H, ArH), 7.47-7.42 (m, 2H, ArH), 7.267.17 (m, 2H, ArH), 7.02 (s, 1H, ArH), 6.83-6.79 (m, 1H, ArH), 5.98 $(\mathrm{s}, 1 \mathrm{H}, \mathrm{CH}), 4.31(\mathrm{~s}, 1 \mathrm{H}, \mathrm{CH}), 4.00-3.98(\mathrm{~m}, 1 \mathrm{H}, \mathrm{CH}), 3.54(\mathrm{~s}, 3 \mathrm{H}$, $\mathrm{NCH}_{3}$ ), 3.03-3.02 (m, 1H, CH), 2.04-2.02 (m, 1H, CH), 1.62-1.48 $\left(\mathrm{m}, 3 \mathrm{H}, \mathrm{CH}_{2}\right), 1.39-1.26\left(\mathrm{~m}, 2 \mathrm{H}, \mathrm{CH}_{2}\right), 1.19-1.12\left(\mathrm{~m}, 2 \mathrm{H}, \mathrm{CH}_{2}\right)$, 0.94-0.84 (m, 3H, $\mathrm{CH}_{2}$ ); ${ }^{13} \mathrm{C}$ NMR (125 MHz, DMSO): $\delta$ 178.1, $142.7,140.3,137.3,133.9,131.3,131.1,131.1,129.5,129.2$, $129.0,129.0,125.3,124.3,122.3,120.2,118.9$, 113.5, 113.0, $111.2,105.7,94.2,71.7,66.3,58.0,46.6,40.9,37.7,31.1,27.8$, 27.4, 24.6, 19.6 .

$2^{\prime}$-(1H-Indol-3-yl)-1'-nitro-1' $\mathbf{2}^{\prime}, \mathbf{4 a}^{\prime}, 5^{\prime}, 6^{\prime}, 7^{\prime}, 8^{\prime}, 8 \mathbf{a}^{\prime}, 9^{\prime}, 9 \mathbf{a}^{\prime}$-decahydrospiro[indoline-3,3'-pyrrolo[1,2-a]indol]-2-one (4m). Yellow solid; 94\% yield; mp 214-216 ${ }^{\circ} \mathrm{C}$; IR (KBr) 741, 1103, 1194, 1341, 1468, 1543, 1620, 1694, 1711, 2930, 3256, $3440 \mathrm{~cm}^{-1}$; HRMS (EI) calcd for $\mathrm{C}_{26} \mathrm{H}_{26} \mathrm{NaN}_{4} \mathrm{O}_{3}[\mathrm{M}+\mathrm{Na}]^{+} 465.1897$, found $465.1894 .{ }^{1} \mathrm{H}$ NMR (600 MHz, DMSO-D $): \delta 10.97$ (d, $J=2.4 \mathrm{~Hz}, 1 \mathrm{H}, \mathrm{NH}$ ), $10.16(\mathrm{~s}, 1 \mathrm{H}, \mathrm{NH}), 7.97-7.63(\mathrm{~m}, 1 \mathrm{H}, \mathrm{ArH}), 7.21(\mathrm{t}, J=6.6 \mathrm{~Hz}, 1 \mathrm{H}$, ArH), 7.09 (d, $J=1.2 \mathrm{~Hz}, 2 \mathrm{H}, \mathrm{ArH}), 7.08-7.07$ (m, 1H, ArH), 6.98$6.95(\mathrm{~m}, 1 \mathrm{H}, \mathrm{ArH}), 6.93-6.90(\mathrm{~m}, 1 \mathrm{H}, \mathrm{ArH}), 6.89-6.86(\mathrm{~m}, 1 \mathrm{H}$, ArH), 6.56 (d, $J=7.8 \mathrm{~Hz}, 1 \mathrm{H}, \mathrm{ArH}), 6.43-6.39$ (m, 1H, CH), 4.83 (d, $J=11.4 \mathrm{~Hz}, 1 \mathrm{H}, \mathrm{CH}), 4.69$ (d, $J=6.6 \mathrm{~Hz}, 1 \mathrm{H}, \mathrm{CH}), 4.06-4.03$ $(\mathrm{m}, 1 \mathrm{H}, \mathrm{CH}), 2.16(\mathrm{~d}, J=5.4 \mathrm{~Hz}, 1 \mathrm{H}, \mathrm{CH}), 1.78-1.77(\mathrm{~m}, 1 \mathrm{H}$, $\mathrm{CH}_{2}$ ), 1.58-1.53 (m, 3H, $\mathrm{CH}_{2}$ ), 1.20-1.17 (m, 3H, $\mathrm{CH}_{2}$ ), 1.05-0.91 (m, 3H, $\left.\mathrm{CH}_{2}\right) ;{ }^{13} \mathrm{C}$ NMR (150 MHz, DMSO): $\delta$ 179.6, 143.6, 136.0, 129.9 , 127.8, 127.2, 125.3, 123.7, 121.6, 121.5, 119.0, 118.9, $111.7,109.7,107.7,91.7,73.8,63.3,59.1,43.3,38.4,34.2,29.2$, 27.3, 24.8, 19.7 .

$2^{\prime}$-(1H-Indol-3-yl)-5-methyl-1'-nitro-1 ${ }^{\prime}, 2^{\prime}, 4 a^{\prime}, 5^{\prime}, 6^{\prime}, 7^{\prime}, 8^{\prime}, 8 a^{\prime}, 9^{\prime}$, 9a'-decahydrospiro[indoline-3,3'-pyrrolo[1,2-a]indol]-2-one (4n). Yellow solid; 91\% yield; mp 189-191 ${ }^{\circ} \mathrm{C}$; IR (KBr) 739, 818, 1045, 1333, 1373, 1493, 1547, 1626, 1690, 1711, 2849, 2932, $3277 \mathrm{~cm}^{-1}$; HRMS (EI) calcd for $\mathrm{C}_{27} \mathrm{H}_{28} \mathrm{NaN}_{4} \mathrm{O}_{3}[\mathrm{M}+\mathrm{Na}]^{+} 479.2054$, found 479.2051. ${ }^{1} \mathrm{H}$ NMR (500 MHz, DMSO-D ${ }_{6}$ ): $\delta 10.93(\mathrm{~s}, 1 \mathrm{H}, \mathrm{NH})$, 10.00 (s, 1H, NH), 7.79 (s, 1H, ArH), 7.61 (d, J=8.0 Hz, 1H, ArH), 7.21-7.18 (m, 2H, ArH), 6.95 (t, $J=15.0 \mathrm{~Hz}, 1 \mathrm{H}, \mathrm{ArH}), 6.86(\mathrm{t}, J=$ $14.5 \mathrm{~Hz}, 2 \mathrm{H}, \mathrm{ArH}), 6.42-6.36(\mathrm{~m}, 1 \mathrm{H}, \mathrm{ArH}), 4.77$ (d, $J=11.5 \mathrm{~Hz}$, $1 \mathrm{H}, \mathrm{CH}), 4.74-4.68$ (m, 1H, CH), 4.08-4.01 (m, 1H, CH), 3.18 (d, J $=5.5 \mathrm{~Hz}, 1 \mathrm{H}, \mathrm{CH}), 2.24\left(\mathrm{~s}, 3 \mathrm{H}, \mathrm{CH}_{3}\right), 2.16-2.13(\mathrm{~m}, 1 \mathrm{H}, \mathrm{CH}), 1.75-$ $1.73(\mathrm{~m}, 1 \mathrm{H}, \mathrm{CH}), 1.56-1.51\left(\mathrm{~m}, 3 \mathrm{H}, \mathrm{CH}_{2}\right), 1.26-1.23\left(\mathrm{~m}, 1 \mathrm{H}, \mathrm{CH}_{2}\right)$, 1.18-1.15 (m, 2H, $\left.\mathrm{CH}_{2}\right), 1.05-1.00\left(\mathrm{~m}, 2 \mathrm{H}, \mathrm{CH}_{2}\right), 0.98-0.92(\mathrm{~m}, 1 \mathrm{H}$, $\left.\mathrm{CH}_{2}\right) ;{ }^{13} \mathrm{C}$ NMR (125 MHz, DMSO): $\delta$ 179.5, 141.1, 136.0, 130.5, 130.0, 127.9, 127.7, 125.3, 123.7, 121.4, 119.1, 118.8, 111.7, 109.4, 107.8, 91.6, 73.8, 63.3, 59.0, 43.6, 38.4, 34.2, 29.2, 27.3, 24.8, 21.2, 19.7.

$2^{\prime}$-(1H-Indol-3-yl)-5-methoxy-1'-nitro-1 ${ }^{\prime}, 2^{\prime}, 4 a^{\prime}, 5^{\prime}, 6^{\prime}, 7^{\prime}, 8^{\prime}, 8 a^{\prime}$, $9^{\prime}, 9 a^{\prime}$-decahydrospiro[indoline-3, $3^{\prime}$-pyrrolo[1,2-a] indol]-2-one (4o). Yellow solid; 95\% yield; mp 178-180 ${ }^{\circ} \mathrm{C}$; IR (KBr) 754, 1032, 1206, 1337, 1456, 1491, 1543, 1707, 2930, $3375 \mathrm{~cm}^{-1}$; HRMS (EI) calcd for $\mathrm{C}_{27} \mathrm{H}_{28} \mathrm{NaN}_{4} \mathrm{O}_{4}[\mathrm{M}+\mathrm{Na}]^{+}$495.2003, found 515, 495.2006. ${ }^{1} \mathrm{H}$ NMR (400 MHz, DMSO-D $)_{6}$ ): $\delta 10.72$ (d, $\left.J=1.2 \mathrm{~Hz} 1 \mathrm{H}, \mathrm{NH}\right), 9.74$ (s, $1 \mathrm{H}, \mathrm{NH}), 7.52$ (d, J=8.0 Hz, 1H, ArH), 7.47 (d, $J=2.4 \mathrm{~Hz}, 1 \mathrm{H}, \mathrm{ArH})$, $6.96(\mathrm{t}, J=10.4 \mathrm{~Hz}, 2 \mathrm{H}, \mathrm{ArH}), 6.72(\mathrm{t}, J=15.2 \mathrm{~Hz}, 1 \mathrm{H}, \mathrm{ArH}), 6.64(\mathrm{t}, J$ $=14.8 \mathrm{~Hz}, 1 \mathrm{H}, \operatorname{ArH}), 6.39(\mathrm{t}, J=10.8 \mathrm{~Hz}, 1 \mathrm{H}, \operatorname{ArH}), 6.19(\mathrm{t}, J=$ $14.0 \mathrm{~Hz}, 1 \mathrm{H}, \mathrm{ArH}), 6.15(\mathrm{t}, J=11.2 \mathrm{~Hz}, 1 \mathrm{H}, \mathrm{CH}), 4.63(\mathrm{~d}, J=$ $11.6 \mathrm{~Hz}, 1 \mathrm{H}, \mathrm{CH}), 4.46-4.39(\mathrm{~m}, 1 \mathrm{H}, \mathrm{CH}), 3.49$ (s, 3H, $\left.\mathrm{OCH}_{3}\right), 2.94$ $(\mathrm{d}, J=4.8 \mathrm{~Hz}, 1 \mathrm{H}, \mathrm{CH}), 1.92-1.88(\mathrm{~m}, 1 \mathrm{H}, \mathrm{CH}), 1.59-1.52(\mathrm{~m}, 1 \mathrm{H}$, $\mathrm{CH}_{2}$ ), 1.31-1.27 (m, 3H, $\mathrm{CH}_{2}$ ), 1.04-0.92 (m, 2H, $\mathrm{CH}_{2}$ ), 0.83-0.71 $\left(\mathrm{m}, 4 \mathrm{H}, \mathrm{CH}_{2}\right) ;{ }^{13} \mathrm{C}$ NMR (100 MHz, DMSO): $\delta$ 179.6, 155.0, 136.9, 135.9, 127.9, 126.5, 123.6, 121.5, 119.2, 118.8, 115.4, 114.2, 111.7, 110.0, 107.9, 91.6, 74.2, 63.3, 58.7, 56.3, 43.0, 38.3, 34.2, 29.2, 27.3, 24.8, 19.7 .

5-Fluoro-2'-(1H-indol-3-yl)-1' -nitro-1 ${ }^{\prime}, 2^{\prime}, 4 a^{\prime}, 5^{\prime}, 6^{\prime}, 7^{\prime}, 8^{\prime}, 8 a^{\prime}, 9^{\prime}$, 9a'-decahydrospiro[indoline-3, $3^{\prime}$-pyrrolo[1,2-a]indol]-2-one (4p). Yellow solid; 92\% yield; mp 151-153 ${ }^{\circ} \mathrm{C}$; IR (KBr) 743, 816, 1180, 1337, 1487, 1545, 1717, 2855, 2930, $3414 \mathrm{~cm}^{-1}$; HRMS (EI) calcd for $\mathrm{C}_{26} \mathrm{H}_{25} \mathrm{NaFN}_{4} \mathrm{O}_{3}[\mathrm{M}+\mathrm{Na}]^{+}$483.1803, found 483.1807. ${ }^{1} \mathrm{H} \mathrm{NMR}$ (500 MHz, DMSO-D $): \delta 10.97(\mathrm{~s}, 1 \mathrm{H}, \mathrm{NH}), 10.17$ (s, 1H, NH), 8.03 $(\mathrm{d}, J=8.0 \mathrm{~Hz}, 1 \mathrm{H}, \mathrm{ArH}), 7.71(\mathrm{~d}, J=7.5 \mathrm{~Hz}, 1 \mathrm{H}, \mathrm{ArH}), 7.23$ (d, $J=$ $7.0 \mathrm{~Hz}, 2 \mathrm{H}, \operatorname{ArH}), 6.98(\mathrm{t}, J=14.5 \mathrm{~Hz}, 1 \mathrm{H}, \operatorname{ArH}), 6.92(\mathrm{t}, J=$ $14.5 \mathrm{~Hz}, 2 \mathrm{H}, \mathrm{ArH}), 6.55-6.52(\mathrm{~m}, 1 \mathrm{H}, \mathrm{ArH}), 6.42-6.38$ (m, 1H, CH), $4.88(\mathrm{~d}, J=11.0 \mathrm{~Hz}, 1 \mathrm{H}, \mathrm{CH}), 4.72-4.67(\mathrm{~m}, 1 \mathrm{H}, \mathrm{CH}), 3.91(\mathrm{~s}, 1 \mathrm{H}$, $\mathrm{CH}), 2.17-2.16(\mathrm{~m}, 1 \mathrm{H}, \mathrm{CH}), 1.81-1.78\left(\mathrm{~m}, 1 \mathrm{H}, \mathrm{CH}_{2}\right), 1.55-1.54$ (m, 3H, $\left.\mathrm{CH}_{2}\right), 1.27-1.18\left(\mathrm{~m}, 2 \mathrm{H}, \mathrm{CH}_{2}\right), 1.10-0.96\left(\mathrm{~m}, 3 \mathrm{H}, \mathrm{CH}_{2}\right)$, 0.57-0.55 (m, 1H, $\mathrm{CH}_{2}$ ); ${ }^{13} \mathrm{C}$ NMR (125 MHz, DMSO): $\delta$ 179.6, 159.2, 157.3, 139.8, 136.0, 127.8, 127.2, 123.7, 121.5, 119.0, 118.9, 116.3, 115.4, 111.8, 110.4, 107.5, 91.3, 74.2, 63.4, 58.8, 43.4, 38.3, $34.2,29.2,27.3,24.8,19.6$. 
7-Chloro-2' -(1H-indol-3-yl)-1'-nitro-1 ${ }^{\prime}, \mathbf{2}^{\prime}, \mathbf{4 a}^{\prime}, \mathbf{5}^{\prime}, \mathbf{6}^{\prime}, \mathbf{7}^{\prime}, \mathbf{8}^{\prime}, \mathbf{8 a}^{\prime}, \mathbf{9}^{\prime}$, 9a'-decahydrospiro[indoline-3,3'-pyrrolo[1,2-a] indol]-2-one (4q). Yellow solid; 93\% yield; mp 195-197 ${ }^{\circ} \mathrm{C}$; IR (KBr) 739, 1182, 1337, 1541, 1620, 1713, 2930, 3426, $3449 \mathrm{~cm}^{-1}$; HRMS (EI) calcd for $\mathrm{C}_{26} \mathrm{H}_{26} \mathrm{ClN}_{4} \mathrm{O}_{3}[\mathrm{M}+\mathrm{H}]^{+}$477.1688, found 477.1685. ${ }^{1} \mathrm{H}$ NMR (500 MHz, DMSO-D $\left.\mathrm{D}_{6}\right): \delta 11.00(\mathrm{~s}, 1 \mathrm{H}, \mathrm{NH}), 10.63(\mathrm{~s}, 1 \mathrm{H}, \mathrm{NH}), 8.01$ (d, $J=7.0 \mathrm{~Hz}, 1 \mathrm{H}, \mathrm{ArH}), 7.63(\mathrm{~d}, J=8.0 \mathrm{~Hz}, 1 \mathrm{H}, \mathrm{ArH}), 7.22(\mathrm{t}, J=$ $9.0 \mathrm{~Hz}, 2 \mathrm{H}, \mathrm{ArH}), 7.16(\mathrm{~d}, J=8.0 \mathrm{~Hz}, 1 \mathrm{H}, \mathrm{ArH}), 6.99-6.94(\mathrm{~m}, 2 \mathrm{H}$, ArH), 6.88 (t, $J=14.5 \mathrm{~Hz}, 1 \mathrm{H}, \mathrm{ArH}), 6.42-6.38(\mathrm{~m}, 1 \mathrm{H}, \mathrm{CH}), 4.86$ (d, $J=11.5 \mathrm{~Hz}, 1 \mathrm{H}, \mathrm{CH}), 4.74-4.68(\mathrm{~m}, 1 \mathrm{H}, \mathrm{CH}), 3.90(\mathrm{~d}, J=$ $5.4 \mathrm{~Hz}, 1 \mathrm{H}, \mathrm{CH}), 2.18-2.14(\mathrm{~m}, 1 \mathrm{H}, \mathrm{CH}), 1.81-1.76\left(\mathrm{~m}, 1 \mathrm{H}, \mathrm{CH}_{2}\right)$, 1.59-1.53 (m, 3H, $\left.\mathrm{CH}_{2}\right), 1.27-1.22\left(\mathrm{~m}, 1 \mathrm{H}, \mathrm{CH}_{2}\right), 1.20-1.15(\mathrm{~m}, 1 \mathrm{H}$, $\left.\mathrm{CH}_{2}\right), 1.09-1.00\left(\mathrm{~m}, 2 \mathrm{H}, \mathrm{CH}_{2}\right), 0.96-0.91\left(\mathrm{~m}, 1 \mathrm{H}, \mathrm{CH}_{2}\right), 0.52-0.49$ (m, $\left.1 \mathrm{H}, \mathrm{CH}_{2}\right) ;{ }^{13} \mathrm{C}$ NMR (125 MHz, DMSO): $\delta 179.5,141.3,136.0$, 130.1, 127.7, 127.2, 126.0, 123.8, 122.9, 121.6, 119.0, 118.9, 113.8, 111.8, 107.4, 91.4, 74.5, 63.4, 59.0, 43.6, 38.3, 34.2, 29.3, 27.3, 24.7, 19.7 .

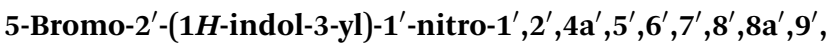
9a'-decahydrospiro[indoline-3, $3^{\prime}$-pyrrolo[1,2-a]indol]-2-one (4r). Yellow solid; $93 \%$ yield; $\mathrm{mp} 192-194{ }^{\circ} \mathrm{C}$; IR (KBr) 739, 820, 1045, 1192, 1333, 1375, 1476, 1545, 1618, 1713, 2851, 2936, 3298, $3333 \mathrm{~cm}^{-1}$; HRMS (EI) calcd for $\mathrm{C}_{26} \mathrm{H}_{25} \mathrm{NaBrN}_{4} \mathrm{O}_{3}[\mathrm{M}+\mathrm{Na}]^{+}$ 543.1002, found 543.1006. ${ }^{1} \mathrm{H}$ NMR (500 MHz, DMSO-D ${ }_{6}$ ): $\delta 10.95(\mathrm{~s}, 1 \mathrm{H}, \mathrm{NH}), 10.95(\mathrm{~s}, 1 \mathrm{H}, \mathrm{NH}), 8.31$ (d, $J=1.5 \mathrm{~Hz}, 1 \mathrm{H}$, $\mathrm{ArH}), 7.65$ (d, $J=8.0 \mathrm{~Hz}, 1 \mathrm{H}, \mathrm{ArH}), 7.25-7.19$ (m, 3H, ArH), 6.98$6.95(\mathrm{~m}, 1 \mathrm{H}, \mathrm{ArH}), 6.89-6.86(\mathrm{~m}, 1 \mathrm{H}, \mathrm{ArH}), 6.48(\mathrm{~d}, J=8.5 \mathrm{~Hz}$, $1 \mathrm{H}, \mathrm{ArH}), 6.49-6.35(\mathrm{~m}, 1 \mathrm{H}, \mathrm{CH}), 4.90(\mathrm{~d}, J=11.5 \mathrm{~Hz}, 1 \mathrm{H}, \mathrm{CH})$, 4.67-4.65 (m, 1H, CH), 4.08-3.90 (m, 1H, CH), $3.18(\mathrm{~d}, J=$ $5.0 \mathrm{~Hz}, 1 \mathrm{H}, \mathrm{CH}), 2.15-2.14\left(\mathrm{~m}, 1 \mathrm{H}, \mathrm{CH}_{2}\right), 1.82-1.81(\mathrm{~m}, 1 \mathrm{H}$, $\left.\mathrm{CH}_{2}\right), 1.54-1.50\left(\mathrm{~m}, 2 \mathrm{H}, \mathrm{CH}_{2}\right), 1.22-1.16\left(\mathrm{~m}, 3 \mathrm{H}, \mathrm{CH}_{2}\right), 1.09-0.97$ (m, 3H, $\left.\mathrm{CH}_{2}\right) ;{ }^{13} \mathrm{C}$ NMR (125 MHz, DMSO): $\delta$ 179.2, 142.9, 136.0, $132.6,130.3,127.8,127.7,123.7,121.5$, 119.1, 118.8, 113.6, 111.8, 111.5, 107.5, 90.9, 74.0, 63.4, 58.8, 43.4, 38.3, 34.2, 29.3, 27.3, 24.8, 19.6.

$2^{\prime}$-(1H-Indol-3-yl)-1', 5-dimethyl-1' ${ }^{\prime}$-nitro-1 $\mathbf{1}^{\prime}, \mathbf{2}^{\prime}, \mathbf{4 a}^{\prime}, \mathbf{5}^{\prime}, \mathbf{6}^{\prime}, \mathbf{7}^{\prime}, \mathbf{8}^{\prime}$, $8 a^{\prime}, 9^{\prime}, 9 a^{\prime}$-decahydrospiro[indoline-3,3'-pyrrolo[1,2-a]indol]-2one (4s). Yellow solid; $89 \%$ yield; mp $193-195{ }^{\circ} \mathrm{C}$; IR (KBr) 741, 814, 1186, 1207, 1493, 1541, 1620, 1728, 2860, 2924, 3292, $3441 \mathrm{~cm}^{-1}$; HRMS (EI) calcd for $\mathrm{C}_{28} \mathrm{H}_{30} \mathrm{NaN}_{4} \mathrm{O}_{3}[\mathrm{M}+\mathrm{Na}]^{+}$ 493.2210, found 493.2206. ${ }^{1} \mathrm{H}$ NMR $\left(500 \mathrm{MHz}\right.$, DMSO-D $\left.{ }_{6}\right)$ : $\delta 11.16(\mathrm{~s}, 1 \mathrm{H}, \mathrm{NH}), 10.33(\mathrm{~s}, 1 \mathrm{H}, \mathrm{NH}), 7.88(\mathrm{~d}, J=7.0 \mathrm{~Hz}, 1 \mathrm{H}$, $\operatorname{ArH}), 7.53(\mathrm{~d}, J=2.0 \mathrm{~Hz}, 1 \mathrm{H}, \mathrm{ArH}), 7.36(\mathrm{~d}, J=8.5 \mathrm{~Hz}, 2 \mathrm{H}, \mathrm{ArH})$, 7.10-7.05 (m, 3H, ArH), $6.71(\mathrm{~d}, J=8.0 \mathrm{~Hz}, 1 \mathrm{H}, \mathrm{ArH}), 4.59(\mathrm{~d}, J=$ $11.0 \mathrm{~Hz}, 1 \mathrm{H}, \mathrm{CH}), 4.34-4.29$ (m, 1H, CH), 3.17 (d, J=3.0 Hz, $1 \mathrm{H}$, $\mathrm{CH}), 2.38\left(\mathrm{~s}, 3 \mathrm{H}, \mathrm{CH}_{3}\right), 2.09-2.05(\mathrm{~m}, 1 \mathrm{H}, \mathrm{CH}), 1.91(\mathrm{~d}, J=$ $\left.12.0 \mathrm{~Hz}, 3 \mathrm{H}, \mathrm{CH}_{3}\right), 1.81-1.75\left(\mathrm{~m}, 1 \mathrm{H}, \mathrm{CH}_{2}\right), 1.57-1.53(\mathrm{~m}, 2 \mathrm{H}$, $\left.\mathrm{CH}_{2}\right), 1.46-1.41\left(\mathrm{~m}, 3 \mathrm{H}, \mathrm{CH}_{2}\right), 1.39\left(\mathrm{~m}, 1 \mathrm{H}, \mathrm{CH}_{2}\right), 1.15(\mathrm{~s}, 3 \mathrm{H}$, $\mathrm{CH}_{2}$ ); ${ }^{13} \mathrm{C}$ NMR (125 MHz, DMSO): $\delta 177.7,140.5,136.4,130.7$, $130.1,128.3,127.9,125.7,124.2,121.4,120.1,119.1,111.8$, 110.2, 107.8, 101.5, 74.5, 66.7, 58.0, 48.7, 40.9, 37.4, 27.9, 27.6, 24.8, 21.4, 20.3, 19.7 .

$\mathbf{2}^{\prime}$-(1H-Indol-3-yl)-5-methoxy-1'-methyl-1'-nitro-1', $\mathbf{2}^{\prime}, \mathbf{4 a}^{\prime}, \mathbf{5}^{\prime}, \mathbf{6}^{\prime}$,

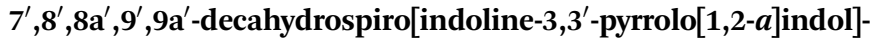
2-one (4t). Yellow solid; 86\% yield; mp $161-163{ }^{\circ} \mathrm{C}$; IR (KBr) 743, 1040, 1201, 1300, 1449, 1487, 1541, 1605, 1728, 2930, 3277, 3418, $3628 \mathrm{~cm}^{-1}$; HRMS (EI) calcd for $\mathrm{C}_{28} \mathrm{H}_{31} \mathrm{~N}_{4} \mathrm{O}_{4}[\mathrm{M}+\mathrm{H}]^{+}$487.2340, found 487.2344. ${ }^{1} \mathrm{H}$ NMR (500 MHz, DMSO-D ${ }_{6}$ ): $\delta 11.17(\mathrm{~s}, 1 \mathrm{H}$, $\mathrm{NH}), 10.29(\mathrm{~s}, 1 \mathrm{H}, \mathrm{NH}), 7.83(\mathrm{~d}, J=8.0 \mathrm{~Hz}, 1 \mathrm{H}, \mathrm{ArH}), 7.53(\mathrm{~d}, J=$ $2.0 \mathrm{~Hz}, 1 \mathrm{H}, \mathrm{ArH}), 7.36(\mathrm{~d}, J=8.0 \mathrm{~Hz}, 1 \mathrm{H}, \mathrm{ArH}), 7.10-7.04(\mathrm{~m}, 3 \mathrm{H}$, $\mathrm{ArH}), 6.89-6.87(\mathrm{~m}, 1 \mathrm{H}, \mathrm{ArH}), 6.75(\mathrm{~d}, J=8.5 \mathrm{~Hz}, 1 \mathrm{H}, \mathrm{ArH}), 4.54$ (d, $J=10.5 \mathrm{~Hz}, 1 \mathrm{H}, \mathrm{CH}), 4.34-4.29(\mathrm{~m}, 1 \mathrm{H}, \mathrm{CH}), 4.06-4.02(\mathrm{~m}$, $1 \mathrm{H}, \mathrm{CH}), 3.80\left(\mathrm{~s}, 3 \mathrm{H}, \mathrm{OCH}_{3}\right), 3.18(\mathrm{~d}, J=4.0 \mathrm{~Hz}, 1 \mathrm{H}, \mathrm{CH}), 2.10-$ $2.06\left(\mathrm{~m}, 1 \mathrm{H}, \mathrm{CH}_{2}\right), 1.90\left(\mathrm{~s}, 3 \mathrm{H}, \mathrm{CH}_{3}\right), 1.79-1.73\left(\mathrm{~m}, 1 \mathrm{H}, \mathrm{CH}_{2}\right)$, 1.56-1.53 (m, 2H, $\left.\mathrm{CH}_{2}\right), 1.46-1.38\left(\mathrm{~m}, 2 \mathrm{H}, \mathrm{CH}_{2}\right), 1.17(\mathrm{~s}, 2 \mathrm{H}$, $\mathrm{CH}_{2}$ ), 1.89-1.94 (m, 2H, $\left.\mathrm{CH}_{2}\right) ;{ }^{13} \mathrm{C}$ NMR (125 MHz, DMSO): $\delta$ 177.5, 154.4, 136.4, 136.3, 128.2, 125.8, 125.5, 121.4, 120.0, 119.1, 115.1, 114.3, 111.9, 110.6, 107.6, 101.5, 74.7, 66.6, 58.1, 56.1, 48.8, 40.9, 37.5, 27.9, 27.6, 24.7, 20.3, 19.7.

5-Fluoro-2'-(1H-indol-3-yl)-1'-methyl-1'-nitro-1' $\mathbf{2}^{\prime}, \mathbf{4 a}^{\prime}, \mathbf{5}^{\prime}, \mathbf{6}^{\prime}, \mathbf{7}^{\prime}$,

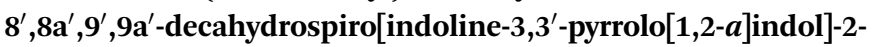
one (4u). Yellow solid; $91 \%$ yield; $\mathrm{mp} 155-157^{\circ} \mathrm{C}$; IR (KBr) 743 , 1184, 1456, 1543, 1730, 2930, 3402, $3437 \mathrm{~cm}^{-1}$; HRMS (EI) calcd for $\mathrm{C}_{27} \mathrm{H}_{27} \mathrm{NaFN}_{4} \mathrm{O}_{3}[\mathrm{M}+\mathrm{Na}]^{+}$497.1959, found 497.1955. ${ }^{1} \mathrm{H}$ (NMR $500 \mathrm{MHz}$, DMSO-D $\mathrm{D}_{6}$ ): $\delta 11.16(\mathrm{~d}, J=1.5 \mathrm{~Hz}, 1 \mathrm{H}, \mathrm{NH}$ ), $10.49(\mathrm{~s}, 1 \mathrm{H}, \mathrm{NH}), 7.92(\mathrm{~d}, J=7.5 \mathrm{~Hz}, 1 \mathrm{H}, \mathrm{ArH}), 7.58-7.56(\mathrm{~m}$, $1 \mathrm{H}, \mathrm{ArH}), 7.53(\mathrm{~d}, J=2.5 \mathrm{~Hz}, 1 \mathrm{H}, \mathrm{ArH}), 7.35(\mathrm{~d}, J=7.0 \mathrm{~Hz}, 1 \mathrm{H}$, ArH), 7.15-7.11 (m, 1H, ArH), 7.09-7.03 (m, 2H, ArH), 6.82-6.80 (m, $1 \mathrm{H}, \mathrm{ArH}), 4.57(\mathrm{~d}, J=11.0 \mathrm{~Hz}, 1 \mathrm{H}, \mathrm{CH}), 4.33-4.32(\mathrm{~m}, 1 \mathrm{H}$, $\mathrm{CH}), 3.17(\mathrm{t}, J=8.0 \mathrm{~Hz}, 1 \mathrm{H}, \mathrm{CH}), 2.50(\mathrm{t}, J=3.5 \mathrm{~Hz}, 1 \mathrm{H}, \mathrm{CH}), 1.89$ (s, 3H, $\left.\mathrm{CH}_{3}\right), 1.57-1.50\left(\mathrm{~m}, 2 \mathrm{H}, \mathrm{CH}_{2}\right), 1.49-1.37\left(\mathrm{~m}, 3 \mathrm{H}, \mathrm{CH}_{2}\right)$, 1.38-1.15 (m, 2H, $\left.\mathrm{CH}_{2}\right), 1.04-0.97\left(\mathrm{~m}, 3 \mathrm{H}, \mathrm{CH}_{2}\right) ;{ }^{13} \mathrm{C}$ NMR $(125$ MHz, DMSO): $\delta$ 177.8, 158.7, 156.8, 139.2, 136.4, 128.2, 125.8, $121.4,120.4,119.0,117.0,116.8,115.4,111.8,111.1,107.6$, 101.7, 74.8, 66.6, 58.1, 48.6, 41.0, 37.1, 27.8, 27.6, 24.7, 20.3, 19.6.

7-Chloro-2'-(1H-indol-3-yl)-1'-methyl-1'-nitro-1' $\mathbf{2}^{\prime}, \mathbf{4 a}^{\prime}, \mathbf{5}^{\prime}, \mathbf{6}^{\prime}, \mathbf{7}^{\prime}$, $8^{\prime}, 8 \mathbf{a}^{\prime}, \mathbf{9}^{\prime}, \mathbf{9 a}^{\prime}$-decahydrospiro[indoline-3,3'-pyrrolo[1,2-a] indol]-2one (4v). Yellow solid; $89 \%$ yield; mp $161-163{ }^{\circ} \mathrm{C}$; IR (KBr) 743 , $1142,1182,1337,1456,1535,1618,1715,2930,3335$, $3401 \mathrm{~cm}^{-1}$; HRMS (EI) calcd for $\mathrm{C}_{27} \mathrm{H}_{28} \mathrm{ClN}_{4} \mathrm{O}_{3}[\mathrm{M}+\mathrm{H}]^{+}$ 491.1844, found 491.1840. ${ }^{1} \mathrm{H}$ NMR (500 MHz, DMSO-D $\left.{ }_{6}\right)$ : $\delta 10.84(\mathrm{~d}, J=1.5 \mathrm{~Hz}, 1 \mathrm{H}, \mathrm{NH}), 10.54(\mathrm{~s}, 1 \mathrm{H}, \mathrm{NH}), 7.45$ (d, $J=$ $7.5 \mathrm{~Hz}, 1 \mathrm{H}, \operatorname{ArH}), 7.30(\mathrm{~d}, J=8.0 \mathrm{~Hz}, 1 \mathrm{H}, \operatorname{ArH}), 7.02(\mathrm{~d}, J=$ $8.0 \mathrm{~Hz}, 1 \mathrm{H}, \mathrm{ArH}), 6.91-6.88(\mathrm{~m}, 2 \mathrm{H}, \mathrm{ArH}), 6.77-6.71(\mathrm{~m}, 1 \mathrm{H}$, ArH), 6.69-6.65 (m, 2H, ArH), 4.23-4.19 (m, 1H, CH), 3.86-3.46 $(\mathrm{m}, 1 \mathrm{H}, \mathrm{ArH}), 3.12(\mathrm{~s}, 1 \mathrm{H}, \mathrm{CH}), 2.94(\mathrm{~d}, J=5.5 \mathrm{~Hz}, 1 \mathrm{H}, \mathrm{CH}), 1.89-$ $1.86\left(\mathrm{~m}, 1 \mathrm{H}, \mathrm{CH}_{2}\right), 1.74\left(\mathrm{~s}, 3 \mathrm{H}, \mathrm{CH}_{3}\right), 1.51-1.47\left(\mathrm{~m}, 1 \mathrm{H}, \mathrm{CH}_{2}\right)$, 1.31-1.26 (m, 3H, $\left.\mathrm{CH}_{2}\right), 1.03-1.00\left(\mathrm{~m}, 1 \mathrm{H}, \mathrm{CH}_{2}\right), 0.94-0.92(\mathrm{~m}$, $\left.2 \mathrm{H}, \mathrm{CH}_{2}\right), 0.82-0.74\left(\mathrm{~m}, 2 \mathrm{H}, \mathrm{CH}_{2}\right) ;{ }^{13} \mathrm{C}$ NMR (125 MHz, DMSO): $\delta$ 180.4, 141.1, 135.5, 130.1, 128.6, 128.4, 124.9, 124.7, 123.1, 121.7, 119.4, 118.3, 113.9, 111.9, 105.6, 100.1, 74.6, 74.2, 58.2, 51.0, 39.0, 36.0, 28.7, 27.6, 25.5, 24.5, 19.4 .

5-Bromo-2'-(1H-indol-3-yl)-1'-methyl-1'-nitro-1' $\mathbf{2}^{\prime}, \mathbf{4 a}^{\prime}, \mathbf{5}^{\prime}, \mathbf{6}^{\prime}, \mathbf{7}^{\prime}$, $8^{\prime}, \mathbf{8 a}^{\prime}, \mathbf{9}^{\prime}, \mathbf{9 a ^ { \prime }}$-decahydrospiro[indoline-3,3'-pyrrolo[1, 2-a] indol]-2one (4w). Yellow solid; 93\% yield; mp $172-174{ }^{\circ} \mathrm{C}$; IR (KBr) 741, 870, 1194, 1344, 1450, 1541, 1616, 1732, 2922, 3287, $3441 \mathrm{~cm}^{-1}$; HRMS (EI) calcd for $\mathrm{C}_{27} \mathrm{H}_{28} \mathrm{BrN}_{4} \mathrm{O}_{3}[\mathrm{M}+\mathrm{H}]^{+}$535.1339, found 535.1352. ${ }^{1} \mathrm{H}$ NMR (500 MHz, DMSO-D ${ }_{6}$ ): $\delta 11.19$ (s, 1H, NH), $10.64(\mathrm{~d}, J=6.0 \mathrm{~Hz}, 1 \mathrm{H}, \mathrm{NH}), 7.87(\mathrm{t}, J=12.5 \mathrm{~Hz}, 1 \mathrm{H}, \mathrm{ArH}), 7.73$ (d, $J=4.5 \mathrm{~Hz}, 1 \mathrm{H}, \mathrm{ArH}), 7.54$ (d, $J=4.0 \mathrm{~Hz}, 1 \mathrm{H}, \mathrm{ArH}), 7.49-7.47$ (m, 1H, ArH), 7.38-7.35 (m, 1H, ArH), 7.10-7.06 (m, 2H, ArH), $6.80(\mathrm{~d}, J=15.0 \mathrm{~Hz}, 1 \mathrm{H}, \mathrm{ArH}), 4.55-4.51(\mathrm{~m}, 1 \mathrm{H}, \mathrm{CH}), 4.34(\mathrm{~s}, 1 \mathrm{H}$, $\mathrm{CH}), 3.17(\mathrm{~s}, 1 \mathrm{H}, \mathrm{CH}), 2.51$ (d, $J=1.0 \mathrm{~Hz}, 1 \mathrm{H}, \mathrm{CH}), 2.09(\mathrm{t}, J=$ 
$\left.10.5 \mathrm{~Hz}, 1 \mathrm{H}, \mathrm{CH}_{2}\right), 1.88$ (t, $\left.J=19.0 \mathrm{~Hz}, 3 \mathrm{H}, \mathrm{CH}_{3}\right), 1.55-1.41(\mathrm{~m}$, $\left.5 \mathrm{H}, \mathrm{CH}_{2}\right), 1.16-1.15\left(\mathrm{~m}, 1 \mathrm{H}, \mathrm{CH}_{2}\right), 1.02-0.96\left(\mathrm{~m}, 3 \mathrm{H}, \mathrm{CH}_{2}\right) ;{ }^{13} \mathrm{C}$ NMR (125 MHz, DMSO): $\delta$ 177.3, 142.3, 136.4, 133.4, 129.7, 128.1, 126.6, 125.9, 121.4, 120.1, 119.1, 113.3, 112.4, 111.9, 107.4, 101.9, 74.5, 66.5, 58.2, 48.9, 41.0, 37.1, 27.8, 27.6, 24.7, 20.3, 19.6.

$2^{\prime}$-(1H-Indol-3-yl)-1'-nitro-1' $\mathbf{2}^{\prime}, \mathbf{5}^{\prime}, 6^{\prime}, 7^{\prime}, 7 \mathbf{a}^{\prime}$-hexahydrospiro [indoline-3, $\mathbf{3}^{\prime}$-pyrrolizin]-2-one (8a). Yellow solid; 96\% yield; $\mathrm{mp}$ 232-234 ${ }^{\circ} \mathrm{C}$; IR (KBr) 754, 1196, 1344, 1545, 1620, 1719, $3352 \mathrm{~cm}^{-1}$; HRMS (EI) calcd for $\mathrm{C}_{22} \mathrm{H}_{20} \mathrm{NaN}_{4} \mathrm{O}_{3}[\mathrm{M}+\mathrm{Na}]^{+}$ 411.1428, found 411.1425. ${ }^{1} \mathrm{H}$ NMR (500 MHz, DMSO-D 6 ): $\delta 10.99$ (s, 1H, NH), $10.23(\mathrm{~s}, 1 \mathrm{H}, \mathrm{NH}), 7.90(\mathrm{~d}, J=7.5 \mathrm{~Hz}, 1 \mathrm{H}$, $\mathrm{ArH}), 7.47$ (d, $J=8.0 \mathrm{~Hz}, 1 \mathrm{H}, \mathrm{ArH}), 7.22$ (t, $J=11.5 \mathrm{~Hz}, 2 \mathrm{H}, \operatorname{ArH})$, $6.95(\mathrm{t}, J=15.5 \mathrm{~Hz}, 1 \mathrm{H}, \mathrm{ArH}), 6.83(\mathrm{t}, J=15.0 \mathrm{~Hz}, 2 \mathrm{H}, \mathrm{ArH}), 6.56$ $(\mathrm{d}, J=8.0 \mathrm{~Hz}, 2 \mathrm{H}, \mathrm{ArH}), 6.25(\mathrm{t}, J=20.0 \mathrm{~Hz}, 1 \mathrm{H}, \mathrm{CH}), 4.91(\mathrm{~d}, J=$ $10.5 \mathrm{~Hz}, 1 \mathrm{H}, \mathrm{CH}), 4.66-4.61(\mathrm{~m}, 1 \mathrm{H}, \mathrm{CH}), 3.43-3.34(\mathrm{~m}, 1 \mathrm{H}$, $\left.\mathrm{CH}_{2}\right), 2.64-2.61\left(\mathrm{~m}, 1 \mathrm{H}, \mathrm{CH}_{2}\right), 2.04-1.99\left(\mathrm{~m}, 1 \mathrm{H}, \mathrm{CH}_{2}\right), 1.96-1.92$ (m, $1 \mathrm{H}, \mathrm{CH}_{2}$ ), 1.69-1.63 (m, 1H, $\left.\mathrm{CH}_{2}\right), 1.45-1.39\left(\mathrm{~m}, 1 \mathrm{H}, \mathrm{CH}_{2}\right)$; ${ }^{13} \mathrm{C}$ NMR (125 MHz, DMSO): $\delta$ 178.6, 143.7, 136.0, 130.1, 127.6, 127.2 , 126.1, 123.8, 121.7, 121.5, 119.0, 118.8, 111.8, 110.0, 107.6, 94.2, 74.7, 63.7, 51.0, 44.1, 27.9, 25.6.

$2^{\prime}$-(1H-Indol-3-yl)-5-methyl-1'-nitro-1 ${ }^{\prime}, 2^{\prime}, 5^{\prime}, 6^{\prime}, 7^{\prime}, 7 \mathbf{a}^{\prime}$-hexahydrospiro[indoline-3,3'-pyrrolizin]-2-one (8b). Yellow solid; $94 \%$ yield; mp 231-233 ${ }^{\circ} \mathrm{C}$; IR (KBr) 743, 812, 1209, 1337, 1493, 1545, 1626, 1717, 2968, $3381 \mathrm{~cm}^{-1}$; HRMS (EI) calcd for $\mathrm{C}_{23} \mathrm{H}_{22} \mathrm{NaN}_{4} \mathrm{O}_{3}[\mathrm{M}+\mathrm{Na}]^{+}$425.1584, found 425.1587. ${ }^{1} \mathrm{H} \mathrm{NMR}$ (500 MHz, DMSO-D 6$): \delta 11.00(\mathrm{~s}, 1 \mathrm{H}, \mathrm{NH}), 10.14(\mathrm{~s}, 1 \mathrm{H}, \mathrm{NH})$, 7.74 (s, 1H, ArH), 7.47 (d, $J=8.0 \mathrm{~Hz}, 1 \mathrm{H}, \operatorname{ArH}), 7.21$ (d, $J=$ $7.5 \mathrm{~Hz}, 2 \mathrm{H}, \operatorname{ArH}), 6.95(\mathrm{t}, J=15.0 \mathrm{~Hz}, 1 \mathrm{H}, \operatorname{ArH}), 6.89$ (d, $J=$ $7.5 \mathrm{~Hz}, 1 \mathrm{H}, \operatorname{ArH}), 6.84(\mathrm{t}, J=14.5 \mathrm{~Hz}, 1 \mathrm{H}, \operatorname{ArH}), 6.44$ (d, $J=$ $7.5 \mathrm{~Hz}, 1 \mathrm{H}, \mathrm{ArH}), 6.23(\mathrm{t}, J=20.0 \mathrm{~Hz}, 1 \mathrm{H}, \mathrm{CH}), 4.87$ (d, $J=$ $10.5 \mathrm{~Hz}, 1 \mathrm{H}, \mathrm{CH}), 4.66-4.61(\mathrm{~m}, 1 \mathrm{H}, \mathrm{CH}), 3.42-3.39(\mathrm{~m}, 1 \mathrm{H}$, $\left.\mathrm{CH}_{2}\right), 2.65-2.63\left(\mathrm{~m}, 1 \mathrm{H}, \mathrm{CH}_{2}\right), 2.27\left(\mathrm{~s}, 3 \mathrm{H}, \mathrm{CH}_{3}\right), 2.02-1.98(\mathrm{~m}$, $\left.1 \mathrm{H}, \mathrm{CH}_{2}\right), 1.94-1.93\left(\mathrm{~m}, 1 \mathrm{H}, \mathrm{CH}_{2}\right), 1.69-1.66\left(\mathrm{~m}, 1 \mathrm{H}, \mathrm{CH}_{2}\right), 1.44-$ $1.40\left(\mathrm{~m}, 1 \mathrm{H}, \mathrm{CH}_{2}\right) ;{ }^{13} \mathrm{C} \mathrm{NMR}(125 \mathrm{MHz}, \mathrm{DMSO}): \delta 178.6,141.2$, $136.1,130.7,130.2,127.9,127.6$, 126.1, 123.8, 121.5, 118.9, 118.8, 111.8, 109.6, 107.6, 94.2, 74.7, 63.7, 51.0, 44.4, 27.9, 25.7, 21.2.

$2^{\prime}$-(1H-Indol-3-yl)-7-methyl-1'-nitro-1 $1^{\prime}, 2^{\prime}, 5^{\prime}, 6^{\prime}, 7^{\prime}, 7 \mathbf{a}^{\prime}$-hexahydrospiro[indoline-3, $\mathbf{3}^{\prime}$-pyrrolizin]-2-one (8c). Yellow solid; 93\% yield; mp $213-216^{\circ} \mathrm{C}$; IR (KBr) 698, 756, 1190, 1341, 1458, 1541, $1719,3360 \mathrm{~cm}^{-1}$; HRMS (EI) calcd for $\mathrm{C}_{23} \mathrm{H}_{22} \mathrm{NaN}_{4} \mathrm{O}_{3}[\mathrm{M}+\mathrm{Na}]^{+}$ 425.1584, found 425.1580. ${ }^{1} \mathrm{H}$ NMR (500 MHz, DMSO-D 6 ): $\delta 11.00(\mathrm{~s}, 1 \mathrm{H}, \mathrm{NH}), 10.31(\mathrm{~s}, 1 \mathrm{H}, \mathrm{NH}), 7.72(\mathrm{~d}, J=7.0 \mathrm{~Hz}, 1 \mathrm{H}$, $\operatorname{ArH}), 7.53$ (d, $J=8.0 \mathrm{~Hz}, 1 \mathrm{H}, \operatorname{ArH}), 6.22$ (d, $J=9.5 \mathrm{~Hz}, 2 \mathrm{H}, \operatorname{ArH})$, $6.96(\mathrm{t}, J=14.0 \mathrm{~Hz}, 1 \mathrm{H}, \mathrm{ArH}), 6.92(\mathrm{~d}, J=7.5 \mathrm{~Hz}, 1 \mathrm{H}, \mathrm{ArH}), 6.87$ $(\mathrm{d}, J=7.0 \mathrm{~Hz}, 2 \mathrm{H}, \mathrm{ArH}), 6.26(\mathrm{t}, J=20.0 \mathrm{~Hz}, 1 \mathrm{H}, \mathrm{CH}), 4.91(\mathrm{~d}, J=$ $10.5 \mathrm{~Hz}, 1 \mathrm{H}, \mathrm{CH}), 4.66-4.62(\mathrm{~m}, 1 \mathrm{H}, \mathrm{CH}), 3.35$ (s, 1H, $\left.\mathrm{CH}_{2}\right), 2.59-$ $2.57\left(\mathrm{~m}, 1 \mathrm{H}, \mathrm{CH}_{2}\right), 2.02-2.00\left(\mathrm{~m}, 1 \mathrm{H}, \mathrm{CH}_{2}\right), 1.99\left(\mathrm{~s}, 3 \mathrm{H}, \mathrm{CH}_{3}\right)$, 1.94-1.92 (m, 1H, $\left.\mathrm{CH}_{2}\right), 1.66-1.64\left(\mathrm{~m}, 1 \mathrm{H}, \mathrm{CH}_{2}\right), 1.44-1.42(\mathrm{~m}$, $1 \mathrm{H}, \mathrm{CH}_{2}$ ); ${ }^{13} \mathrm{C}$ NMR (125 MHz, DMSO): $\delta$ 179.1, 142.3, 136.0, $131.4,127.7,125.7,124.4,123.8,121.7,121.6,119.2,119.0$, 118.8, 111.8, 107.8, 94.6, 74.8, 63.5, 51.0, 43.8, 27.9, 25.6, 16.7.

$2^{\prime}$-(1H-Indol-3-yl)-5-methoxy-1'-nitro-1' $\mathbf{2}^{\prime}, 5^{\prime}, 6^{\prime}, 7^{\prime}, 7 \mathbf{a}^{\prime}$-hexahydrospiro[indoline-3,3' ${ }^{\prime}$-pyrrolizin]-2-one (8d). Yellow solid; 92\% yield; mp 217-220 ${ }^{\circ} \mathrm{C}$; IR (KBr) 689, 752, 1028, 1209, 1342, 1493, 1539, 1607, 1718, 2953, $3389 \mathrm{~cm}^{-1}$; HRMS (EI) calcd for $\mathrm{C}_{23} \mathrm{H}_{23} \mathrm{~N}_{4} \mathrm{O}_{4}[\mathrm{M}+\mathrm{H}]^{+}$419.1714, found 419.1718. ${ }^{1} \mathrm{H}$ NMR (500
MHz, DMSO-D 6 ): $\delta 11.00(\mathrm{~s}, 1 \mathrm{H}, \mathrm{NH}), 10.08(\mathrm{~s}, 1 \mathrm{H}, \mathrm{NH}), 7.62(\mathrm{~d}, J$ $=8.0 \mathrm{~Hz}, 2 \mathrm{H}, \operatorname{ArH}), 7.22(\mathrm{~d}, J=6.5 \mathrm{~Hz}, 2 \mathrm{H}, \operatorname{ArH}), 6.96(\mathrm{t}, J=$ $14.5 \mathrm{~Hz}, 1 \mathrm{H}, \operatorname{ArH}), 6.86(\mathrm{t}, J=15.0 \mathrm{~Hz}, 1 \mathrm{H}, \operatorname{ArH}), 6.66$ (d, $J=$ $8.0 \mathrm{~Hz}, 1 \mathrm{H}, \operatorname{ArH}), 6.47(\mathrm{~d}, J=8.5 \mathrm{~Hz}, 1 \mathrm{H}, \operatorname{ArH}), 6.26(\mathrm{t}, J=$ $20.0 \mathrm{~Hz}, 1 \mathrm{H}, \mathrm{CH}), 4.93$ (d, J=11.0 Hz, 1H, CH), 4.64-4.59 (m, $1 \mathrm{H}, \mathrm{CH}), 3.74\left(\mathrm{~s}, 3 \mathrm{H}, \mathrm{OCH}_{3}\right), 3.48-3.43\left(\mathrm{~m}, 1 \mathrm{H}, \mathrm{CH}_{2}\right), 2.64-2.61$ $\left(\mathrm{m}, 1 \mathrm{H}, \mathrm{CH}_{2}\right), 2.02-1.93\left(\mathrm{~m}, 2 \mathrm{H}, \mathrm{CH}_{2}\right), 1.67-1.64\left(\mathrm{~m}, 1 \mathrm{H}, \mathrm{CH}_{2}\right)$, 1.48-1.42 (m, 1H, $\mathrm{CH}_{2}$ ); ${ }^{13} \mathrm{C}$ NMR (125 MHz, DMSO): $\delta$ 178.6, $155.0,136.9,136.0,127.7,127.3$, 123.8, 121.5, 119.0, 118.9, $115.3,114.3,111.8,110.2,107.7,94.1,75.1,63.6,56.3,50.8,43.8$, $27.9,25.7$.

5-Fluoro-2'-(1H-indol-3-yl)-1'-nitro- $1^{\prime}, 2^{\prime}, 5^{\prime}, 6^{\prime}, 7^{\prime}, 7 a^{\prime}$-hexahydrospiro[indoline-3, $3^{\prime}$-pyrrolizin]-2-one (8e). Yellow solid; 93\% yield; mp 223-226 ${ }^{\circ} \mathrm{C}$; IR (KBr) 754, 1188, 1341, 1485, 1541, $1721,3375 \mathrm{~cm}^{-1}$; HRMS (EI) calcd for $\mathrm{C}_{22} \mathrm{H}_{19} \mathrm{NaFN}_{4} \mathrm{O}_{3}[\mathrm{M}+\mathrm{Na}]^{+}$ 429.1333, found 429.1330. ${ }^{1} \mathrm{H}$ NMR (500 MHz, DMSO-D 6 ): $\delta 11.02$ (s, 1H, NH), 10.28 (s, 1H, NH), 7.97 (d, $J=9.0 \mathrm{~Hz}, 1 \mathrm{H}$, $\operatorname{ArH}), 7.59$ (d, $J=7.5 \mathrm{~Hz}, 1 \mathrm{H}, \operatorname{ArH}), 7.22$ (d, $J=10.0 \mathrm{~Hz}, 2 \mathrm{H}, \operatorname{ArH}$ ), 6.98-6.92 (m, 2H, ArH), 6.87 (t, $J=15.0 \mathrm{~Hz}, 1 \mathrm{H}, \mathrm{ArH}), 6.55-6.53$ $(\mathrm{m}, 1 \mathrm{H}, \mathrm{ArH}), 6.27(\mathrm{t}, J=20.0 \mathrm{~Hz}, 1 \mathrm{H}, \mathrm{CH}), 4.95(\mathrm{~d}, J=10.5 \mathrm{~Hz}$, $1 \mathrm{H}, \mathrm{CH}), 4.65-4.60$ (m, $1 \mathrm{H}, \mathrm{CH}), 3.47-3.42$ (m, $\left.1 \mathrm{H}, \mathrm{CH}_{2}\right), 2.66-$ $2.63\left(\mathrm{~m}, 1 \mathrm{H}, \mathrm{CH}_{2}\right), 2.02-1.94\left(\mathrm{~m}, 2 \mathrm{H}, \mathrm{CH}_{2}\right), 1.69-1.65(\mathrm{~m}, 1 \mathrm{H}$, $\left.\mathrm{CH}_{2}\right)$, 1.48-1.40 (m, 1H, $\left.\mathrm{CH}_{2}\right) ;{ }^{13} \mathrm{C}$ NMR (125 MHz, DMSO): $\delta 178.7,159.1,157.2,139.9,136.0,127.6,123.8,121.7,119.0$, 118.8, 116.5, 116.3, 115.5, 111.8, 110.5, 107.4, 93.7, 75.0, 63.7, $50.9,44.0,27.9,25.7$.

$2^{\prime}$-(1H-Indol-3-yl)-7-methyl-1'-nitro-1 $\mathbf{1}^{\prime}, 2^{\prime}, 5^{\prime}, 6^{\prime}, 7^{\prime}, 7 \mathbf{a}^{\prime}$-hexahydrospiro[indoline-3, $\mathbf{3}^{\prime}$-pyrrolizin]-2-one (8f). Yellow solid; 92\% yield; mp 112-114 ${ }^{\circ} \mathrm{C}$; IR (KBr) 744, 1140, 1341, 1506, 1549, 1638, 1724, 2972, $3418 \mathrm{~cm}^{-1}$; HRMS (EI) calcd for $\mathrm{C}_{22} \mathrm{H}_{19} \mathrm{~F}_{2} \mathrm{~N}_{4} \mathrm{O}_{3}$ $[\mathrm{M}+\mathrm{H}]^{+}$425.1420, found 425.1425. ${ }^{1} \mathrm{H}$ NMR (400 MHz, DMSO$\left.\mathrm{D}_{6}\right): \delta 11.04(\mathrm{~d}, J=1.2 \mathrm{~Hz}, 1 \mathrm{H}, \mathrm{NH}), 10.39(\mathrm{~s}, 1 \mathrm{H}, \mathrm{NH}), 8.31-8.27$ (m, 1H, ArH), 7.61 (d, $J=8.0 \mathrm{~Hz}, 1 \mathrm{H}, \operatorname{ArH}), 7.24(\mathrm{~d}, J=8.4 \mathrm{~Hz}$, $2 \mathrm{H}, \mathrm{ArH}), 6.98(\mathrm{t}, J=14.8 \mathrm{~Hz}, 1 \mathrm{H}, \operatorname{ArH}), 6.89(\mathrm{t}, J=14.8 \mathrm{~Hz}, 1 \mathrm{H}$, ArH), 6.61-6.56 (m, 1H, ArH), 6.27 (t, J=20.0 Hz, 1H, CH), 4.95 $(\mathrm{d}, J=10.8 \mathrm{~Hz}, 1 \mathrm{H}, \mathrm{CH}), 4.63-4.57(\mathrm{~m}, 1 \mathrm{H}, \mathrm{CH}), 3.47-3.41(\mathrm{~m}$, $\left.1 \mathrm{H}, \mathrm{CH}_{2}\right), 2.69-2.63\left(\mathrm{~m}, 1 \mathrm{H}, \mathrm{CH}_{2}\right), 2.03-1.92\left(\mathrm{~m}, 2 \mathrm{H}, \mathrm{CH}_{2}\right), 1.70-$ $1.63\left(\mathrm{~m}, 1 \mathrm{H}, \mathrm{CH}_{2}\right), 1.46-1.43\left(\mathrm{~m}, 1 \mathrm{H}, \mathrm{CH}_{2}\right) ;{ }^{13} \mathrm{C} \mathrm{NMR}(100 \mathrm{MHz}$, DMSO): $\delta$ 179.0, 140.5, 140.4, 136.0, 127.5, 123.8, 122.1, 121.6, 119.0, 118.8, 117.6, 117.4, 111.9, 107.2, 99.8, 99.6, 93.3, 74.7, 63.6, 50.8, 43.8, 28.0, 25.6.

6-Chloro-2'-(1H-indol-3-yl)-1'-nitro-1' ${ }^{\prime} 2^{\prime}, 5^{\prime}, 6^{\prime}, 7^{\prime}, 7 \mathbf{a}^{\prime}$-hexahydrospiro[indoline-3, $\mathbf{3}^{\prime}$-pyrrolizin]-2-one $(\mathbf{8 g})$. Yellow solid; 95\% yield; mp 217-219 ${ }^{\circ} \mathrm{C}$; IR (KBr) 754, 926, 1074, 1194, 1339, 1454, 1541, 1618, 1726, 2976, $3347 \mathrm{~cm}^{-1}$; HRMS (EI) calcd for $\mathrm{C}_{22} \mathrm{H}_{19} \mathrm{NaClN}_{4} \mathrm{O}_{3}[\mathrm{M}+\mathrm{Na}]^{+}$445.1038, found 445.1042. ${ }^{1} \mathrm{H} \mathrm{NMR}$ (400 MHz, DMSO-D $): \delta 11.06(\mathrm{~s}, 1 \mathrm{H}, \mathrm{NH}), 10.46(\mathrm{~s}, 1 \mathrm{H}, \mathrm{NH})$, $8.01(\mathrm{~d}, J=8.0 \mathrm{~Hz}, 1 \mathrm{H}, \mathrm{ArH}), 7.53$ (d, $J=8.0 \mathrm{~Hz}, 1 \mathrm{H}, \mathrm{ArH}), 7.24$ $(\mathrm{t}, J=7.2 \mathrm{~Hz}, 2 \mathrm{H}, \operatorname{ArH}), 7.02-6.96(\mathrm{~m}, 2 \mathrm{H}, \operatorname{ArH}), 6.87(\mathrm{t}, J=$ $14.8 \mathrm{~Hz}, 1 \mathrm{H}, \operatorname{ArH}), 6.59$ (d, $J=2.0 \mathrm{~Hz}, 1 \mathrm{H}, \operatorname{ArH}), 6.27(\mathrm{t}, J=$ $20.4 \mathrm{~Hz}, 1 \mathrm{H}, \mathrm{CH}), 4.94(\mathrm{~d}, J=10.8 \mathrm{~Hz}, 1 \mathrm{H}, \mathrm{CH}), 4.65-4.59(\mathrm{~m}$, $1 \mathrm{H}, \mathrm{CH}), 2.63\left(\mathrm{t}, J=14.0 \mathrm{~Hz}, 1 \mathrm{H}, \mathrm{CH}_{2}\right), 2.06-1.93\left(\mathrm{~m}, 3 \mathrm{H}, \mathrm{CH}_{2}\right)$, 1.72-1.63 (m, 1H, $\left.\mathrm{CH}_{2}\right), 1.48-1.39\left(\mathrm{~m}, 1 \mathrm{H}, \mathrm{CH}_{2}\right) ;{ }^{13} \mathrm{C} \mathrm{NMR}(100$ MHz, DMSO): $\delta$ 178.6, 145.2, 136.0, 134.5, 128.9, 127.5, 125.0, 123.9, 121.6, 121.4, 119.0, 118.7, 111.9, 110.1, 107.3, 93.7, 74.4, 63.6, 51.0, 43.8, 27.9, 25.6. 
7-Chloro-2'-(1H-indol-3-yl)-1'-nitro-1' $\mathbf{2}^{\prime}, \mathbf{5}^{\prime}, \mathbf{6}^{\prime}, \mathbf{7}^{\prime}, \mathbf{7 a}^{\prime}$-hexahydrospiro[indoline-3,3'-pyrrolizin]-2-one (8h). Yellow solid; 93\% yield; mp 180-182 ${ }^{\circ} \mathrm{C}$; IR (KBr) 741, 1142, 1177, 1337, 1458, 1547, 1620, 1726, 2976, 3372, $3424 \mathrm{~cm}^{-1}$; HRMS (EI) calcd for $\mathrm{C}_{22} \mathrm{H}_{20} \mathrm{ClN}_{4} \mathrm{O}_{3}[\mathrm{M}+\mathrm{H}]^{+}$423.1218, found $423.1213 .{ }^{1} \mathrm{H}$ NMR $(400$ MHz, DMSO-D $\left.{ }_{6}\right): \delta 11.07$ (s, 1H, NH), $10.75(\mathrm{~s}, 1 \mathrm{H}, \mathrm{NH}), 7.98(\mathrm{~d}$, $J=7.6 \mathrm{~Hz}, 1 \mathrm{H}, \mathrm{ArH}), 7.53$ (d, $J=8.0 \mathrm{~Hz}, 1 \mathrm{H}, \mathrm{ArH}), 7.27-7.19(\mathrm{~m}$, $3 \mathrm{H}, \mathrm{ArH}), 7.02-6.96(\mathrm{~m}, 2 \mathrm{H}, \mathrm{ArH}), 6.86(\mathrm{t}, J=14.8 \mathrm{~Hz}, 1 \mathrm{H}, \mathrm{ArH})$, $6.28(\mathrm{~m}, 1 \mathrm{H}, \mathrm{CH}), 4.76(\mathrm{~d}, J=10.4 \mathrm{~Hz} 1 \mathrm{H}, \mathrm{CH}), 4.67-4.61(\mathrm{~m}, 1 \mathrm{H}$, $\mathrm{CH}), 2.61\left(\mathrm{~d}, J=14.0 \mathrm{~Hz}, 1 \mathrm{H}, \mathrm{CH}_{2}\right), 2.07-2.02\left(\mathrm{~m}, 1 \mathrm{H}, \mathrm{CH}_{2}\right)$, 2.00-1.93 (m, 2H, $\left.\mathrm{CH}_{2}\right), 1.71-1.48\left(\mathrm{~m}, 1 \mathrm{H}, \mathrm{CH}_{2}\right), 1.48-1.42(\mathrm{~m}$, $\left.1 \mathrm{H}, \mathrm{CH}_{2}\right) ;{ }^{13} \mathrm{C}$ NMR (100 MHz, DMSO): $\delta$ 178.5, 141.4, 136.0, 130.2, 127.9, 127.5, 126.1, 123.9, 123.0, 121.6, 119.1, 118.7, 114.1, 111.9, 107.3, 93.9, 75.3, 63.6, 50.9, 44.0, 27.9, 25.6.

6-Bromo-2'-(1H-indol-3-yl)-1'-nitro-1 ${ }^{\prime}, \mathbf{2}^{\prime}, \mathbf{5}^{\prime}, \mathbf{6}^{\prime}, \mathbf{7}^{\prime}, 7 \mathrm{a}^{\prime}$-hexahydrospiro[indoline-3,3'-pyrrolizin]-2-one (8i). Yellow solid; 91\% yield; $\mathrm{mp} 190-192{ }^{\circ} \mathrm{C}$; IR (KBr) 745, 914, 1126, 1337, 1543, 1612, $1724,2974,3418 \mathrm{~cm}^{-1}$; HRMS (EI) calcd for $\mathrm{C}_{22} \mathrm{H}_{19} \mathrm{NaBrN}_{4} \mathrm{O}_{3}[\mathrm{M}$ $+\mathrm{Na}]^{+}$489.0533, found 489.0537. ${ }^{1} \mathrm{H}$ NMR (400 MHz, DMSO-D ${ }_{6}$ ): $\delta 11.05(\mathrm{~s}, 1 \mathrm{H}, \mathrm{NH}), 10.43(\mathrm{~s}, 1 \mathrm{H}, \mathrm{NH}), 7.95(\mathrm{~d}, J=8.0 \mathrm{~Hz}, 1 \mathrm{H}$, $\mathrm{ArH}), 7.53(\mathrm{~d}, J=8.0 \mathrm{~Hz}, 1 \mathrm{H}, \mathrm{ArH}), 7.25-7.23(\mathrm{~m}, 2 \mathrm{H}, \mathrm{ArH}), 7.15$ $(\mathrm{t}, J=9.2 \mathrm{~Hz}, 1 \mathrm{H}, \mathrm{ArH}), 6.98(\mathrm{t}, J=15.2 \mathrm{~Hz}, 1 \mathrm{H}, \mathrm{ArH}), 6.87(\mathrm{~m}$, $1 \mathrm{H}, \mathrm{ArH}), 6.72(\mathrm{~d}, J=1.6 \mathrm{~Hz}, 1 \mathrm{H}, \mathrm{ArH}), 6.26(\mathrm{t}, J=20.0 \mathrm{~Hz}, 1 \mathrm{H}$, $\mathrm{CH}), 4.93(\mathrm{~d}, J=10.8 \mathrm{~Hz}, 1 \mathrm{H}, \mathrm{CH}), 4.65-4.58(\mathrm{~m}, 1 \mathrm{H}, \mathrm{CH}), 2.63(\mathrm{t}$, $\left.J=14.0 \mathrm{~Hz}, 1 \mathrm{H}, \mathrm{CH}_{2}\right), 2.05-1.91\left(\mathrm{~m}, 3 \mathrm{H}, \mathrm{CH}_{2}\right), 1.70-1.63(\mathrm{~m}, 1 \mathrm{H}$, $\left.\mathrm{CH}_{2}\right)$, 1.48-1.41 (m, $\left.1 \mathrm{H}, \mathrm{CH}_{2}\right) ;{ }^{13} \mathrm{C}$ NMR (100 MHz, DMSO): $\delta 178.5,145.3,136.0,129.2,127.5,125.4,124.3,123.9,123.0$, 121.6, 119.0, 118.7, 112.8, 111.9, 107.3, 93.7, 74.5, 63.6, 51.0, 43.8, 27.9, 25.6.

7-Bromo-2'-(1H-indol-3-yl)-1'-nitro-1' $\mathbf{2}^{\prime}, 5^{\prime}, 6^{\prime}, 7^{\prime}, 7 \mathbf{a}^{\prime}$-hexahydrospiro[indoline-3,3'-pyrrolizin]-2-one (8j). Yellow solid; 95\% yield; $\mathrm{mp} 155-157{ }^{\circ} \mathrm{C}$; IR (KBr) 741, 1138, 1179, 1337, 1456, 1545, 1618, 1724, 2972, $3414 \mathrm{~cm}^{-1}$; HRMS (EI) calcd for $\mathrm{C}_{22} \mathrm{H}_{20} \mathrm{BrN}_{4} \mathrm{O}_{3}[\mathrm{M}+\mathrm{H}]^{+}$467.0713, found 467.0709. ${ }^{1} \mathrm{H}$ NMR (400 MHz, DMSO-D 6 ): $\delta 11.07$ (s, 1H, NH), $10.62(\mathrm{~s}, 1 \mathrm{H}, \mathrm{NH}), 8.01$ (d, $=7.2 \mathrm{~Hz}, 1 \mathrm{H}, \mathrm{ArH}), 7.54(\mathrm{~d}, J=8.0 \mathrm{~Hz}, 1 \mathrm{H}, \operatorname{ArH}), 7.32(\mathrm{~d}, J=$ $8.4 \mathrm{~Hz}, 1 \mathrm{H}, \mathrm{ArH}), 7.25(\mathrm{~m}, 2 \mathrm{H}, \mathrm{ArH}), 6.99(\mathrm{~d}, J=7.2 \mathrm{~Hz}, 1 \mathrm{H}, \mathrm{ArH})$, 6.96-6.91 (m, 1H, ArH), $6.86(\mathrm{t}, J=14.8 \mathrm{~Hz}, 1 \mathrm{H}, \mathrm{ArH}), 6.27(\mathrm{t}, J=$ $20.0 \mathrm{~Hz}, 1 \mathrm{H}, \mathrm{ArH}), 4.95(\mathrm{~d}, J=10.8 \mathrm{~Hz}, 1 \mathrm{H}, \mathrm{CH}), 4.66-4.60(\mathrm{~m}$, $1 \mathrm{H}, \mathrm{CH}), 2.60(\mathrm{t}, J=14.0 \mathrm{~Hz}, 1 \mathrm{H}, \mathrm{CH}), 2.06-2.01\left(\mathrm{~m}, 1 \mathrm{H}, \mathrm{CH}_{2}\right)$, 1.98-1.92 (m, $\left.2 \mathrm{H}, \mathrm{CH}_{2}\right), 1.73-1.64\left(\mathrm{~m}, 1 \mathrm{H}, \mathrm{CH}_{2}\right), 1.47-1.43(\mathrm{~m}$, $\left.1 \mathrm{H}, \mathrm{CH}_{2}\right) ;{ }^{13} \mathrm{C}$ NMR $(100 \mathrm{MHz}, \mathrm{DMSO}): \delta 178.4,143.1,136.0$, 133.1, 127.8, 127.6, 126.5, 123.9, 123.4, 121.6, 119.0, 118.7, 111.9, 107.3, 102.2, 93.9, 75.4, 63.6, 50.9, 43.9, 28.0, 25.6.

$6^{\prime}$-(1H-Indol-3-yl)-7'-nitro-1 $\mathbf{1}^{\prime}, \mathbf{6}^{\prime}, \mathbf{7}^{\prime}, 7 \mathrm{a}^{\prime}$-tetrahydro-3' $H$-spiro [indoline-3,5'-pyrrolo[1,2-c] thiazol]-2-one (8k). Yellow solid; $89 \%$ yield; mp $252-254{ }^{\circ} \mathrm{C}$; IR (KBr) 679, 737, 750, 1196, 1339, 1369, 1472, 1549, 1618, 1715, 3248, $3401 \mathrm{~cm}^{-1}$; HRMS (EI) calcd for $\mathrm{C}_{21} \mathrm{H}_{18} \mathrm{NaN}_{4} \mathrm{O}_{3} \mathrm{~S}[\mathrm{M}+\mathrm{Na}]^{+}$429.0992, found 429.0997. ${ }^{1} \mathrm{H}$ NMR (500 MHz, DMSO-D $\left.{ }_{6}\right): \delta 11.04(\mathrm{~s}, 1 \mathrm{H}, \mathrm{NH}), 9.98(\mathrm{~s}, 1 \mathrm{H}, \mathrm{NH})$, $7.86(\mathrm{~d}, J=7.0 \mathrm{~Hz}, 1 \mathrm{H}, \mathrm{ArH}), 7.32$ (d, $J=2.5 \mathrm{~Hz}, 1 \mathrm{H}, \mathrm{ArH}), 7.26-$ $7.22(\mathrm{~m}, 2 \mathrm{H}, \mathrm{ArH}), 7.15(\mathrm{t}, J=15 \mathrm{~Hz}, 1 \mathrm{H}, \mathrm{ArH}), 6.94-6.90(\mathrm{~m}, 1 \mathrm{H}$, ArH), 6.67-6.63 (m, 2H, CH), 6.54 (d, $J=7.5 \mathrm{~Hz}, 1 \mathrm{H}, \mathrm{ArH}), 6.47-$ $6.43(\mathrm{~m}, 1 \mathrm{H}, \mathrm{CH}), 4.65(\mathrm{~d}, J=11.5 \mathrm{~Hz}, 1 \mathrm{H}, \mathrm{CH}), 4.52-4.48(\mathrm{~m}$, $1 \mathrm{H}, \mathrm{CH}), 4.05\left(\mathrm{t}, J=16.5 \mathrm{~Hz}, 1 \mathrm{H}, \mathrm{CH}_{2}\right), 3.82(\mathrm{~d}, J=10.0 \mathrm{~Hz}, 1 \mathrm{H}$, $\left.\mathrm{CH}_{2}\right), 3.12-3.08\left(\mathrm{~m}, 1 \mathrm{H}, \mathrm{CH}_{2}\right), 2.97\left(\mathrm{t}, J=19.0 \mathrm{~Hz}, 1 \mathrm{H}, \mathrm{CH}_{2}\right) ;{ }^{13} \mathrm{C}$
NMR (125 MHz, DMSO): $\delta$ 177.6, 143.6, 136.1, 130.7, 127.3, $126.2,125.8,124.4,122.3,121.4,118.9,118.2,111.8,110.3$, 106.7, 86.9, 74.5, 68.0, 55.0, 47.1, 33.4.

6-Chloro-6'-(1H-indol-3-yl)-7'-nitro-1' $\mathbf{6}^{\prime}, \mathbf{6}^{\prime}, \mathbf{7}^{\prime}, 7 \mathrm{a}^{\prime}$-tetrahydro$3^{\prime} H$-spiro[indoline-3,5'-pyrrolo[1,2-c]thiazol]-2-one (81). Yellow solid; $88 \%$ yield; mp $190-192{ }^{\circ} \mathrm{C}$; IR (KBr) 752, 1074, 1128, 1323, 1549, 1612, 1726, $3352 \mathrm{~cm}^{-1}$; HRMS (EI) calcd for $\mathrm{C}_{21} \mathrm{H}_{17} \mathrm{NaClN}_{4} \mathrm{O}_{3} \mathrm{~S}[\mathrm{M}+\mathrm{Na}]^{+}$463.0602, found 463.0606. ${ }^{1} \mathrm{H}$ NMR (500 MHz, DMSO- $\left.\mathrm{D}_{6}\right): \delta 11.07(\mathrm{~s}, 1 \mathrm{H}, \mathrm{NH}), 10.13(\mathrm{~s}, 1 \mathrm{H}, \mathrm{NH})$, $7.91(\mathrm{~d}, J=8.0 \mathrm{~Hz}, 1 \mathrm{H}, \mathrm{ArH}), 7.33(\mathrm{~d}, J=2.5 \mathrm{~Hz}, 1 \mathrm{H}, \mathrm{ArH}), 7.26-$ $7.21(\mathrm{~m}, 1 \mathrm{H}, \mathrm{ArH}), 7.19(\mathrm{~d}, J=2.0 \mathrm{~Hz}, 1 \mathrm{H}, \mathrm{ArH}), 6.96-6.93(\mathrm{~m}$, $1 \mathrm{H}, \operatorname{ArH}), 6.72(\mathrm{~d}, J=6.5 \mathrm{~Hz}, 2 \mathrm{H}, \mathrm{ArH}), 6.55(\mathrm{~d}, J=1.5 \mathrm{~Hz}, 1 \mathrm{H}$, ArH), 6.47-6.43 (m, 1H, CH), 4.67 (d, J=11.5 Hz, 1H, CH), 4.52$4.47(\mathrm{~m}, 1 \mathrm{H}, \mathrm{CH}), 4.06\left(\mathrm{~d}, J=10.0 \mathrm{~Hz}, 1 \mathrm{H}, \mathrm{CH}_{2}\right), 3.82(\mathrm{~d}, J=$ $\left.10.0 \mathrm{~Hz}, 1 \mathrm{H}, \mathrm{CH}_{2}\right), 3.12-3.08\left(\mathrm{~m}, 1 \mathrm{H}, \mathrm{CH}_{2}\right), 3.00(\mathrm{t}, J=19.0 \mathrm{~Hz}$, $\left.1 \mathrm{H}, \mathrm{CH}_{2}\right) ;{ }^{13} \mathrm{C}$ NMR (125 MHz, DMSO): $\delta$ 177.6, 145.2, 136.2, $135.0,127.9,127.2,124.7,124.6,122.0,121.5,119.0,118.1$, $111.9,110.3,106.4,86.6,74.2,68.1,55.1,46.9,33.4$.

\section{Conflicts of interest}

There are no conflicts to declare.

\section{Acknowledgements}

This work was supported by National Natural Science Foundation of China (No. 31700179), Applied Basic Research Project of Yunnan (Nos. 2017FD073, 2017FD156), Scientific Research Foundation of Yunnan Provincial Education Department (No. 2017ZZX075) and PhD research startup foundation of Yunnan Normal University (Nos. 150025, 160056). We are grateful to Dr Yu-Xin Yan and Xiao-Di Hong (School of Vocational and Technical Education, Yunnan Normal University) for their assistance in the characterized experiment of IR spectra and melting points.

\section{Notes and references}

1 (a) R. S. Varma in Green chemistry: challenging perspectives, ed. P. Tundo and P. T. Anastas, Oxford University Press, Oxford, 2000, pp. 221-244; (b) P. Anastas and N. Eghbali, Chem. Soc. Rev., 2010, 39, 301-312.

2 (a) S. L. Y. Tang, R. L. Smith and M. Poliakoff, Green Chem., 2005, 7, 761-762; (b) C. Capello, U. Fischer and K. Hungerbühler, Green Chem., 2007, 9, 927-934; (c) P. G. Jessop, Green Chem., 2011, 13, 1391-1398.

3 (a) R. S. Varma, Green Chem., 2008, 10, 1129-1130; (b) M. B. Gawande, V. D. B. Bonifácio, R. Luque, P. S. Branco and R. S. Varma, Chem. Soc. Rev., 2013, 42, 5522-5551.

4 (a) M. Khoobi, T. M. Delshad, M. Vosooghi, M. Alipour, H. Hamadi, E. Alipour, M. P. Hamedani, Z. Safaei, A. Foroumadi and A. Shafiee, J. Magn. Magn. Mater., 2015, 375, 217-226; (b) A. Khazaei, M. A. Zolfigol, F. Karimitabar, I. Nikokar and A. R. Moosavi-Zare, RSC Adv., 2015, 5, 71402-71412; (c) Y.-L. Ma, K.-M. Wang, R. Huang, J. Lin and S.-J. Yan, Green Chem., 2017, 19, 3574-3584; (d) H. Wu, X.-M. Chen, Y. Wan, L. Ye, H.-Q. Xin, H.-H. Xu, C.-H. Yue, 
L.-L. Pang, R. Ma and D.-Q. Shi, Tetrahedron Lett., 2009, 50, 1062-1065; (e) L.-J. Yan, J.-L. Wang, D. Xu, K. S. Burgess, A.-F. Zhu, Y.-Y. Rao, X.-B. Chen and Y.-C. Wang, ChemistrySelect, 2018, 3, 662-665.

5 (a) C. M. R. Volla, I. Atodiresei and M. Rueping, Chem. Rev., 2014, 114, 2390-2431; (b) A. Dömling, W. Wang and K. Wang, Chem. Rev., 2012, 112, 3083-3135; (c) B. M. Trost and A. J. Frontier, J. Am. Chem. Soc., 2000, 122, 11727-11728; (d) B. M. Trost, A. C. Gutierrez and R. C. Livingston, Org. Lett., 2009, 11, 2539-2542; (e) B. Jiang, S.-J. Tu, P. Kaur, W. Wever and G.-G. Li, J. Am. Chem. Soc., 2009, 131, 1166011661; (f) B. Jiang, M.-S. Yi, F. Shi, S.-J. Tu, S. Pindi, P. McDowell and G. Li, Chem. Commun., 2012, 48, 808-810; (g) J. Sun, Y. Sun, H. Gong, Y.-J. Xie and C.-G. Yan, Org. Lett., 2012, 14, 5172-5175; (h) X. Feng, Q. Wang, W. Lin, G.-L. Dou, Z.-B. Huang and D.-Q. Shi, Org. Lett., 2013, 15, 2542-2545.

6 (a) C. de Graaff, E. Ruijte and R. V. A. Orru, Chem. Soc. Rev., 2012, 41, 3969-4009; (b) P. Slobbe, E. Ruijte and R. V. A. Orru, MedChemComm, 2012, 3, 1189-1218.

7 (a) C.-B. Cui, H. Kakeya and H. Osada, J. Antibiot., 1996, 49, 832-835; (b) H. Conroy and J. K. Chakrabarti, Tetrahedron Lett., 1959, 1, 6-13; (c) F. M. Lovell, R. Pepinsky and A. J. C. Wilson, Tetrahedron Lett., 1959, 1, 1-5.

8 (a) N. R. Ball-Jones, J. J. Badillo and A. K. Franz, Org. Biomol. Chem., 2012, 10, 5165-5181; (b) C. B. Cui, H. Kakeya and H. Osada, Tetrahedron, 1996, 52, 12651-12666; (c) J. Leclercq, M. C. de Pauw-Gillet, R. Bassleer and L. Angenot, J. Ethnopharmacol., 1986, 15, 305-316.

9 (a) K. Ding, Y.-P. Lu, Z. Nikolovska-Coleska, S. Qiu, Y.-S. Ding, W. Gao, J. Stuckey, K. Krajewski, P. P. Roller, Y. Tomita, D. A. Parrish, J. R. Deschamps and S.-M. Wang, J. Am. Chem. Soc., 2005, 127, 10130-10131; (b) S. Rana, E. C. Blowers, C. Tebbe, J. I. Contreras, P. Radhakrishnan, S. Kizhake, T. Zhou, R. N. Rajule, J. L. Arnst, A. R. Munkarah, R. Rattan and A. Natarajan, J. Med. Chem., 2016, 59, 5121-5127; (c) R. F. George, N. S. M. Ismail, J. Stawinski and A. S. Girgis, Eur. J. Med. Chem., 2013, 68, 339-351; (d) Y. Arun, K. Saranraj, C. Balachandran and P. T. Perumal, Eur. J. Med. Chem., 2014, 74, 50-64.

10 (a) A. Thangamani, Eur. J. Med. Chem., 2010, 45, 6120-6126; (b) D. Kathirvelan, J. Haribabu, B. S. R. Reddy, C. Balachandran and V. Duraipandiyan, Bioorg. Med. Chem. Lett., 2015, 25, 389-399; (c) S. U. Maheswari, K. Balamurugan, S. Perumal, P. Yogeeswari and D. Sriram, Bioorg. Med. Chem. Lett., 2010, 20, 7278-7282; (d) R. R. Kumar, S. Perumal, P. Senthilkumar, P. Yogeeswar and D. Sriram, J. Med. Chem., 2008, 51, 5731-5735; (e) A. Nandakumar, P. Thirumurugan, P. T. Perumal, P. Vembu, M. N. Ponnuswamy and P. Ramesh, Bioorg. Med. Chem. Lett., 2010, 20, 4252-4258.

11 B. K. S. Yeung, B. Zou, M. Rottmann, S. B. Lakshminarayana, S. H. Ang, S. Y. Leong, J. Tan, J. Wong, S. Keller-Maerki, C. Fischli, A. Goh, E. K. Schmitt, P. Krastel, E. Francotte, K. Kuhen, D. Plouffe, K. Henson, T. Wagner, E. A. Winzeler, F. Petersen, R. Brun, V. Dartois,
T. T. Diagana and T. H. Keller, J. Med. Chem., 2010, 53, 5155-5164.

12 R. Murugan, S. Anbazhagan and S. S. Narayanan, Eur. J. Med. Chem., 2009, 44, 3272-3279.

13 (a) P. Prasanna, K. Balamurugan, S. Perumal, P. Yogeeswari and D. Sriram, Eur. J. Med. Chem., 2010, 45, 5653-5661; (b) R. S. Kumar, S. M. Rajesh, S. Perumal, D. Banerjee, P. Yogeeswari and D. Sriram, Eur. J. Med. Chem., 2010, 45, 411-422.

14 G. Kumari, M. Modi, S. K. Gupta and R. K. Singh, Eur. J. Med. Chem., 2011, 46, 1181-1188.

15 N. Karalı, Ö. Güzel, N. Özsoy, S. Özbey and A. Salman, Eur. J. Med. Chem., 2010, 45, 1068-1077.

16 (a) S.-Y. Li, J. M. Finefield, J. D. Sunderhaus, T. J. Mcafoos, R. M. Williams and D. H. Sherman, J. Med. Chem., 2012, 134, 788-791; (b) S. Crosignani, C. Jorand-Lebrun, P. Page, G. Campbell, V. Colovray, M. Missotten, Y. Humbert, C. Cleva, J.-F. Arrighi, M. Gaudet, Z. Johnson, P. Ferro and A. Chollet, J. Med. Chem., 2011, 2, 644-649; (c) K. Karthikeyan, P. M. Sivakumar, M. Doble and P. T. Perumal, Eur. J. Med. Chem., 2010, 45, 3446-3452.

17 (a) P. B. Alper, C. Meyers, A. Lerchner, D. R. Siegel and E. M. Carreira, Angew. Chem., Int. Ed., 1999, 38, 3186-3189; (b) C. V. Galliford and K. A. Scheidt, Angew. Chem., Int. Ed., 2007, 46, 8748-8758.

18 (a) J.-Y. Li, A. Corma and J.-H. Yu, Chem. Soc. Rev., 2015, 44, 7112-7127; (b) J.-J. Feng, T.-Y. Lin, C.-Z. Zhu, H.-M. Wang, H.-H. Wu and J.-L. Zhang, J. Am. Chem. Soc., 2016, 138, 2178-2181; (c) X.-X. Guo, D.-W. Gu, Z.-X. Wu and W.-B. Zhang, Chem. Rev., 2014, 115, 1622-1651; (d) J.-J. Feng and J.-L. Zhang, ACS Catal., 2016, 6, 6651-6661; (e) M. Bakthadoss and N. Sivakumar, Synlett, 2009, 6, 1014-1018; (f) M. Bakthadoss, N. Sivakumar, A. Devaraj and D. S. Sharada, Synthesis, 2011, 13, 2136-2146.

19 (a) K. Ding, Y.-P. Lu, Z. Nikolovska-Coleska, G.-P. Wang, S. Qiu, S. Shangary, W. Gao, D.-G. Qin, J. Stuckey, K. Krajewski, P. P. Roller and S. Wang, J. Med. Chem., 2006, 49, 3432-3435; (b) V. V. Vintonyak, K. Warburg, H. Kruse, S. Grimme, K. Hübel, D. Rauth and H. Waldmann, Angew. Chem., Int. Ed., 2010, 49, 5902-5905; (c) A. Fensome, W. R. Adams, A. L. Adams, T. J. Berrodin, J. Cohen, C. Huselton, A. Illenberger, J. C. Karen, M. A. Hudak, A. G. Marella, E. G. Melenski, C. C. McComas, C. A. Mugford, O. D. Slayeden, M. Yudt, J. Zhang, P. Zhang, Y. Zhu, R. C. Winneker and J. E. Wrobel, J. Med. Chem., 2008, 51, 1861-1873.

20 (a) K. Debnath, K. Singha and A. Pramanik, RSC Adv., 2015, 5, 31866-31877; (b) C. A. Maier and B. Wüensch, J. Med. Chem., 2002, 45, 438-448; (c) G. Lang, A. Pinkert, J. W. Blunt and M. H. G. Munro, J. Nat. Prod., 2005, 68, 1796-1798; (d) C. Macleod, B. I. Martinez-Teipel, W. M. Barker and R. E. Dolle, J. Comb. Chem., 2006, 8, 132-140.

21 H.-Y. Wang and D.-Q. Shi, ACS Comb. Sci., 2013, 15, 261-266. 22 (a) B. B. Touré and D. G. Hall, Chem. Rev., 2009, 109, 44394486; (b) S. M. Paul, D. S. Mytelka, C. T. Dunwiddie, C. C. Persinger, B. H. Munos, S. R. Lindborg and 
A. L. Schacht, Nat. Rev. Drug Discovery, 2010, 9, 203-214; (c) Y. Arun, G. Bhaskar, C. Balachandran, S. Ignacimuthu and P. T. Perumal, Bioorg. Med. Chem. Lett., 2013, 23, 1839-1845. 23 (a) G. Chen, Y.-Q. Miao, R. Zhou, L. Zhang, J. Zhang and X.-J. Hao, Res. Chem. Intermed., 2013, 39, 2445-2450; (b) R. T. Pardasani, P. Pardasani, V. Chaturvedi, S. K. Yadav, A. Saxena and I. Sharma, Heteroat. Chem., 2003, 14, 36-41; (c) S. N. Singh, S. Regati, A. K. Paul, M. Layek, S. Jayaprakash, K. V. Reddy, G. S. Deora, S. Mukherjee and M. Pal, Tetrahedron Lett., 2013, 54, 5448-5452; (d) G. P. Rizzi, J. Org. Chem., 1970, 35, 2069-2070.

24 (a) P. R. Mali, L. C. Rao, V. M. Bangade, P. K. Shirsat, S. A. George, N. Jaqadeesh babu and H. M. Meshram, New J. Chem., 2016, 40, 2225-2232; (b) S. Haddad, S. Boudriga, T. N. Akhaja, J. P. Raval, F. Porzio, A. Soldera, M. Askri,
M. Knorr, Y. Rousselin, M. M. Kubicki and D. Rajani, New J. Chem., 2015, 39, 520-528.

25 (a) S. M. Rajesh, S. Perumal, J. C. Menéndez, P. Yogeeswari and D. Sriram, MedChemComm, 2011, 2, 626-630; (b) A. Y. Barkov, N. S. Zimnitskiy, V. Y. Korotaev, I. B. Kutyashev, V. S. Moshkin and V. Y. Sosnovskikh, Tetrahedron, 2016, 72, 6825-6836; (c) S. Kanchithalaivan, M. A. Rani and R. R. Kumar, Synth. Commun., 2014, 44, 3122-3129.

26 (a) D. Xu, J.-L. Wang, L.-J. Yan, M.-Q. Yuan, X.-T. Xie and Y.-C. Wang, Tetrahedron: Asymmetry, 2016, 27, 1121-1132; (b) Y.-C. Wang, D. Li, J. Lin and K. Wei, RSC Adv., 2015, 5, 5863-5874; (c) Y.-C. Wang, S. Ji, K. Wei and J. Lin, RSC Adv., 2014, 4, 30850-30856. 